\title{
Encoding Intensity in Ventral Cochlear Nucleus Following Acoustic Trauma: Implications for Loudness Recruitment
}

\author{
Shanoing CaI, ${ }^{1,2}$ Wei-Li D. Ma, ${ }^{1}$ and Eric D. Young ${ }^{1}$ \\ ${ }^{1}$ Center for Hearing and Balance and Department of Biomedical Engineering, The Johns Hopkins University, Baltimore, \\ MD 21205, USA \\ ${ }^{2}$ Harvard-MIT Division of Health Science and Technology and Speech Communication Group, Research Laboratory of \\ Electronics, Massachusetts Institute of Technology, 36-579, 50 Vassar Street, Cambridge, MA 02139, USA
}

Received: 23 February 2008; Accepted: 23 September 2008; Online publication: 15 October 2008

\begin{abstract}
Loudness recruitment, an abnormally rapid growth of perceived loudness with sound level, is a common symptom of sensorineural hearing loss. Following acoustic trauma, auditory-nerve rate responses are reduced, and rate grows more slowly with sound level, which seems inconsistent with recruitment (Heinz et al., J. Assoc. Res. Otolaryngol. 6:91-105, 2005). However, rate-level functions (RLFs) in the central nervous system may increase in either slope or saturation value following trauma (e.g., Salvi et al., Hear. Res. 147:261-274, 2000), suggesting that recruitment may arise from central changes. In this paper, we studied RLFs of neurons in ventral cochlear nucleus (VCN) of the cat after acoustic trauma. Trauma did not change the general properties of VCN neurons, and the usual VCN functional classifications remained valid (chopper, primary-like, onset, etc.). After trauma, non-primary-like neurons, most noticeably choppers, exhibited elevated maximum discharge rates and steeper RLFs for frequencies at and near best frequency (BF). Primary-like neurons showed the opposite changes. To relate the neurons' responses to recruitment, rate-balance functions were computed; these show the sound level required to give equal rates in a normal and a traumatized ear and are analogous to loudness balance functions that show the sound levels giving equal perceptual loudness in the two ears of a monaurally hearing-impaired person. The ratebalance functions showed recruitment-like steepening
\end{abstract}

Correspondence to: Eric D. Young · Center for Hearing and Balance and Department of Biomedical Engineering · The Johns Hopkins University·Baltimore, MD 21205, USA. email: eyoung@jhu.edu of their slopes in non-primary-like neurons in all conditions. However, primary-like neurons showed recruitment-like behavior only when rates were summated across neurons of all BFs. These results suggest that the non-primary-like, especially chopper, neurons may be the most peripheral site of the physiological changes in the brain that underlie recruitment.

Keywords: ventral cochlear nucleus, acoustic trauma, sensorineural hearing loss, loudness recruitment, hyperacusis, auditory nerve, neuroplasticity, sound intensity, sound level, neural encoding, rate-level function, bushy cell, stellate cell, primary-like units, chopper units

\section{INTRODUCTION}

Loudness recruitment is an abnormal relationship between sound intensity and perceived loudness that may accompany sensorineural hearing loss (SNHL; Moore 2002). In this condition, the audibility threshold is elevated, but a given increase in intensity above threshold causes significantly greater than normal increase in loudness. The rate of loudness growth in the impaired ear is often so steep that, at high intensities (60-100 dB SPL, e.g., Stillman et al. 1993), the loudness in the impaired ear matches that in the normal one. Loudness recruitment has been primarily studied in humans (e.g., Miskolczy-Fodor 1960; Hellman and Zwislocki 1981; Moore et al. 1985; Zeng and Turner 1991) and has also been demonstrated in behavioral animals with SNHL (Pugh et al. 1979). 
The physiological mechanisms of loudness recruitment are not well understood. In this study, we assume that overall sound intensity or loudness is encoded by the discharge rates of auditory nerve (AN) fibers (Sachs and Abbas 1974; Smith 1988; Palmer and Evans 1982; Winter et al. 1990). One hypothesis of the physiological origin of loudness recruitment is that the rate-level functions (RLFs) of AN fibers are steeper in SNHL, as was observed with ototoxic damage (e.g., Harrison 1981). This hypothesis is consistent with the observation that the velocity-intensity relationship of the basilar membrane becomes steeper after the loss of outer-hair cell (OHC) amplification (Ruggero and Rich 1991). However, with acoustic trauma (AT), OHC loss can be accompanied by inner hair cell damage (Liberman and Dodds 1984), which compromises cochlear transduction and lowers the firing rates of AN fibers (Liberman and Kiang 1984; Sewell 1984). As a result, the slopes of their RLFs become shallower on average (e.g., Salvi et al. 1983; Heinz and Young 2004). In cats following AT, the summed RLFs of AN fibers over either a narrow or wide range of best frequencies (BFs) surrounding the stimulus frequency do not show abnormally steep slopes, a result inconsistent with loudness recruitment (Heinz et al. 2005).

Adding to this paradoxical situation is the hyperactivity seen in central auditory neurons after SNHL, which is characterized by an abnormally rapid increase of response with stimulus intensity or by a heightened maximum response or both (e.g., Salvi et al. 2000; Langers et al. 2007). This central hyperactivity qualitatively resembles loudness recruitment and thus is a potential neural correlate. It has been suggested that central hyperactivity reflects a homeostatic plasticity, which aims at maintaining a relatively constant level of neural activity in the face of reduced AN inputs (Schaette and Kempter 2006). However, there are a number of unanswered questions, including at which stage(s) of the central pathway the plastic changes occur, in which populations of neurons, and how the changes interact with peripheral changes to produce enhanced central responses. The relationships between neuronal types and alterations in response properties after permanent SNHL have not been systematically mapped out in central auditory neurons. Most previous studies used evoked potential recordings (e.g., Saunders et al. 1972; Salvi et al. 1990), which did not allow identification of the neuronal sources of changes.

This paper describes the RLFs of single neurons in the ventral cochlear nucleus (VCN) of cats following AT. Neurons recorded in VCN can be classified into several types based on temporal discharge patterns in response to tones. These neuronal types differ in morphology and function (reviewed by Young and
Oertel 2004). In this study, we analyze RLFs summed over various populations of VCN neurons and show that the responses of non-primary-like types of neurons, especially choppers, show changes in rate responses consistent with loudness recruitment, whereas primarylike neurons do not, except in cases where spread of excitation in the population is included.

\section{MATERIALS AND METHODS}

\section{Acoustic trauma}

The animal protocols in this study were approved by the Johns Hopkins University Animal Care and Use Committee (Protocol number CA05M257). Twentyone adult male cats obtained from Liberty Labs were divided into an AT group (12 animals) and a normalhearing control group (nine). Before sound exposure, the animal was checked to have clear outer and middle ears with no signs of infection. The animal was anesthetized with ketamine $(35-45 \mathrm{mg} / \mathrm{kg}$, i.m.) and xylazine $(0.5-0.7 \mathrm{mg} / \mathrm{kg}$, i.m.), supplemented with half-sized doses to maintain an areflexive state, and exposed to a narrowband noise $(50 \mathrm{~Hz}$ bandwidth centered at $2 \mathrm{kHz}, 111-112 \mathrm{~dB}$ SPL) for $4 \mathrm{~h}$.

A recovery period between 33 and 121 days (median, 37 days) was allowed between the exposure and the neural recording. The length of the recovery was not an intended variable in this experiment, but three animals had recoveries over 40 days $(60,72$, and 121 days) because of scheduling problems. There was a tendency for RLFs to be less non-monotonic in these animals, suggesting reduced inhibition, but it was statistically significant $\left(\chi^{2}=10.1, P=0.04,4 d f\right)$ only for the primary-like neurons. There were no significant changes in RLF slopes. The data from these three animals were grouped together with other data in the analyses described below.

\section{Surgical preparation and CAP audiograms}

For neural recording, the animal was anesthetized with ketamine and xylazine as above. Atropine (0.03 $\mathrm{mg} \mathrm{kg}^{-1} \mathrm{day}^{-1}$, i.m.) and dexamethasone $(1 \mathrm{mg} / \mathrm{kg}, \mathrm{i} . \mathrm{m}$.) were given, and a trachea tube was inserted. Body temperature was maintained at $38.5^{\circ} \mathrm{C}$, and hydration was maintained with an i.v. drip of lactated Ringer's solution. Aspiration under visual control was used to make a complete section of the brain between the superior colliculus and the thalamus. Anesthesia was discontinued after the completion of the decerebration. After the experiment, the animal was euthanized by i.v. injection of sodium pentobarbital ( $\sim 800 \mathrm{mg}$ to effect).

The bulla was vented through an $\sim 1$-m length of PE-90 (or similar) tubing. Two types of speakers were 
used in the closed acoustic system: an electrostatic speaker (levels up to $\sim 100 \mathrm{~dB}$ SPL) and a dynamic speaker (up to $\sim 130 \mathrm{~dB}$ SPL). In situ calibration was performed with a calibrated probe microphone (B\&K 4134) placed $2 \mathrm{~mm}$ from the tympanic membrane. The amplitude-frequency responses of both speakers varied less than $\pm 5 \mathrm{~dB}$ in the frequency range between 0.2 and $20 \mathrm{kHz}$. An unexpected acoustic noise originating from the power amplifier driving the dynamic speaker (Crown D-75A, Crown Int. Inc., Elkhart, IL) was discovered after it had been used in experiments on six deafened animals. Measurements indicated that the acoustic noise had a nearly flat spectrum spanning 0.3 to $4 \mathrm{kHz}$ and a spectrum level of $-7.7 \mathrm{~dB}$ re $20 \mu \mathrm{Pa} / \sqrt{ } \mathrm{Hz}$. All neurons recorded from the six animals had thresholds for broadband noise (BBN) at least $20 \mathrm{~dB}$ above this spectrum level, except for three neurons, whose BBN thresholds were 5$10 \mathrm{~dB}$ above the level of the speaker noise. These three neurons were discarded and not used in the analysis. The dynamic speaker was not used after the discovery of this noise.

Before the beginning of single-neuron recording in the VCN, a compound action potential (CAP) audiogram was constructed as a measure of AN thresholds. The CAP stimuli were 10-ms tone pips with 1-ms riseand-fall times repeated at a rate of $10 \mathrm{~Hz}$. Frequencies approximately logarithmically spaced between 0.2 and $20 \mathrm{kHz}$ were presented. For frequencies up to $3 \mathrm{kHz}$, the amplitude of the CM was reduced by reversing the phase of the tone on alternating presentations and by using band-stop digital filtering (Cai 2007). Threshold was interpolated from measurements at three consecutive near-threshold levels ( $5 \mathrm{~dB}$ steps), as the level needed for a CAP amplitude of two standard deviations of the background electrode signal plus its mean. The CAP audiograms of noise-exposed animals showed substantial threshold elevations at frequencies below $6-16 \mathrm{kHz}$, as shown in Figure 1A. At higher frequencies, threshold shifts were smaller or nonexistent. The edge frequency of the audiogram of an exposed animal was defined as the logarithmic center frequency of the right-most segment in the downsloping portion of the audiogram (i.e., at the upper frequency edge of the threshold shift).

\section{Single-neuron recording and classification}

For recording, a portion of the cerebellum was aspirated to expose the dorsal surface of the cochlear nucleus. Custom-made glass-coated platinum-iridium electrodes were used to record from VCN neurons. The electrodes were aligned in the parasaggital plane and lowered into the VCN directly or through part of the DCN under visual control. After a single neuron was isolated, its $\mathrm{BF}$ and threshold were determined,
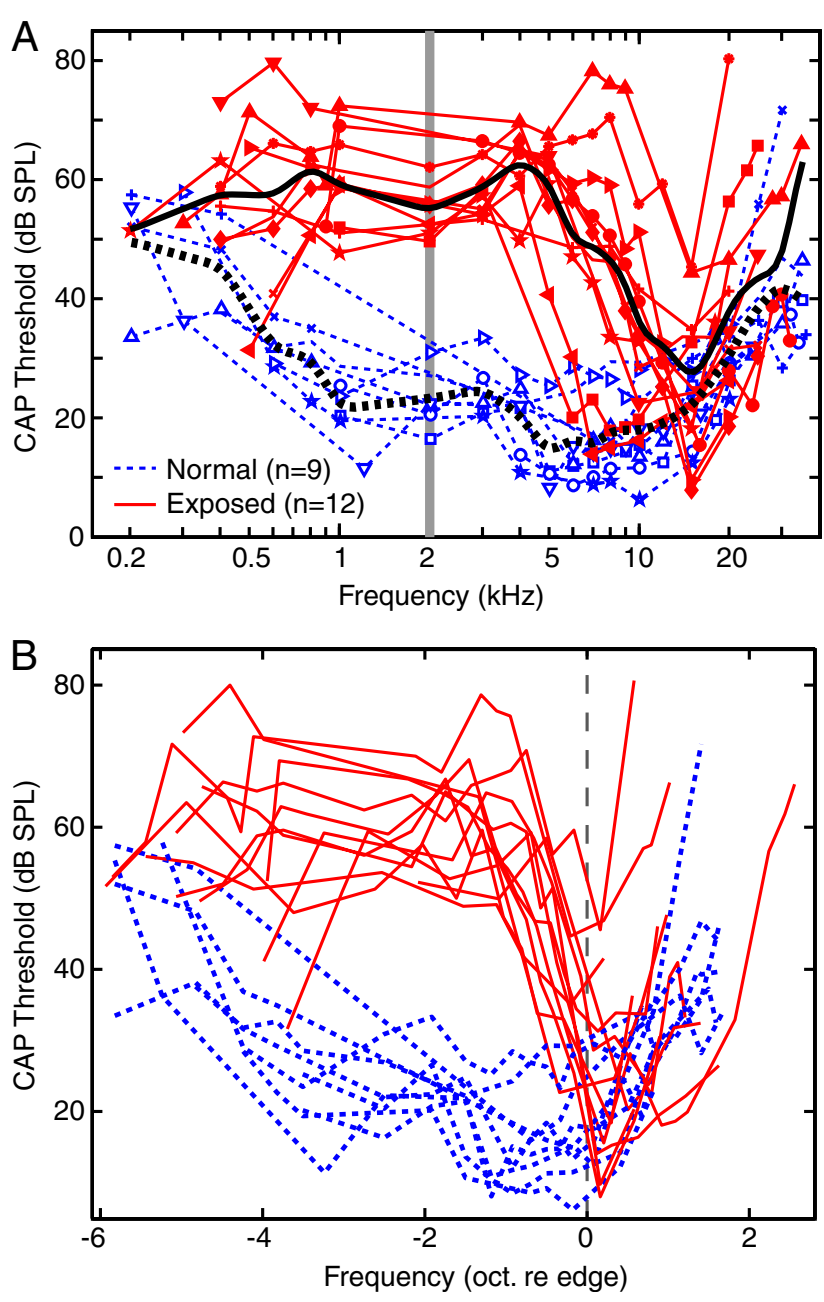

FIG. 1. CAP audiograms in the normal and noise-exposed experiments. A The light lines show CAP audiograms from individual experiments, and the heavy lines are averages for the normal (dashed lines) and exposed (solid lines) animals. The vertical bar shows the frequency of the noise exposure. B CAP audiograms from individual experiments shifted to align their edge frequencies with the geometric mean value $(11.3 \mathrm{kHz})$. Audiograms from normal animals are also aligned at $11.3 \mathrm{kHz}$

usually by constructing a tuning curve as the sound level required to produce a one spike increase in rate in response to a 50-ms tone burst (Kiang et al. 1970). To account for the downward shift of apparent BFs in neurons with substantial threshold shifts and broadened tuning, BFs were set at the low-frequency edge of the high-slope high-frequency portion of the tuning curves of those neurons (Liberman 1984). Tuning quality was estimated as usual by the $10-\mathrm{dB}$ quality factor $(\mathrm{Q} 10)$, defined as the $\mathrm{BF}$ divided by the width of the tuning curve $10 \mathrm{~dB}$ above threshold.

Responses to 50-ms BF-tone bursts (5-ms rise-fall ramps, repeated at $4 / \mathrm{s}$ ) at 30 (and sometimes also 20) $\mathrm{dB}$ above threshold were acquired and a peri-stimulus time histogram (PSTH) was constructed. VCN neurons in both unexposed and exposed ears were 
classified into primary-like (Pri), primary-like-withnotch (PriN), chopper (Ch), onset (On), locker (Lckr), and unusual (Unsl) types, according to the shapes and quantitative measures of the PSTHs (Rhode and Smith 1986; Young et al. 1988); the classification was based on the decision tree given by Blackburn and Sachs (1989). Regularity of discharge and first-spike latency also differed consistently among the response types and were used as an aid to classification. The Pri and PriN neurons are sometimes grouped together into a single primary-like category, called PL below. Three subgroups of choppers were defined (Ch-S, Ch-T, and Ch-L), as in Bourk (1976) and Blackburn and Sachs (1989). Data from the subgroups are not discussed separately in this paper because they did not differ in the measures used; the results from these subclasses were combined in the average measures discussed below and reported as a single class of choppers.

Pri PSTHs are characterized by a relatively high firing rate near onset and a gradual and smooth rate decrease (adaptation). PriN neurons share these properties except for a brief cessation of firing (a notch) immediately following the onset peak. Chopper neurons exhibit multimodal PSTH shapes near the onset of the stimulus, which reflect the regular discharge (chopping) in these neurons. Onset neurons are characterized by a high instantaneous firing rate at short latencies after stimulus onset followed by little or no sustained firing (rate $<100$ spikes/s). Locker neurons are low-BF $(<1 \mathrm{kHz})$ neurons whose PSTHs cannot be classified in the above categories because of strong phase-locking. Unusual-type neurons show PSTHs that cannot be described by any of the three above-mentioned features and/or cannot satisfy the contingencies in the Blackburn-Sachs decision tree to reach a response type. For example, a few neurons with primary-like PSTH shapes showed extraordinarily long first-spike latencies and were categorized as unusual. In some neurons, PSTH data were not obtained; these are listed as "unknown."

\section{Stimuli for rate analysis}

After the completion of PSTH recording, the following stimuli were presented over a range of sound levels in 1-dB ascending steps: (1) tones at the BF of the neuron and at 1 and $2 \mathrm{kHz}$; (2) tones with frequencies spaced at 0.4-octave intervals around the BF (e.g., $-6.0,-5.6,-5.2, \ldots, 3.6$ octaves); (3) BBN with an approximately flat spectrum between 0.2 and $50 \mathrm{kHz}$. The above stimuli had 200-ms durations and $10-\mathrm{ms}$ rise/fall ramps and were spaced by $800-\mathrm{ms}$ silent intervals. The range of sound levels extended from below the rate threshold to either the maximum level of the sound delivery system or the maximum level at which single-neuron isolation was unaffected by neurophonic responses (typically $80-100 \mathrm{~dB}$ SPL for frequencies between 0.5 and $6 \mathrm{kHz}$ ). Each level was repeated one to three times.

\section{Data analysis}

The counting window for calculating driven firing rates was between $10 \mathrm{~ms}$ post-stimulus onset to $10 \mathrm{~ms}$ post-stimulus offset, except for onset neurons, for which the counting window extended from stimulus onset to offset. The 10-ms delays were used to compensate for neural latencies, given a 10-ms stimulus rise time, plus the time delays in our acoustic system. Rates were computed as the number of spikes during the counting window divided by its duration; rates were averaged over all repetitions of each stimulus level and were smoothed (5-dB triangular window) before subsequent calculations. The threshold of a RLF was determined as the first of five consecutive sound levels at which the discharge rates rose above or fell below the $50 \%$ confidence interval for spontaneous rate (SR) based on a Poisson-counts assumption.

The slopes of the rising portion of RLFs were calculated two ways: (1) as the chord slope from threshold to the rate $20 \mathrm{~dB}$ above threshold and (2) using a spline fit to the RLF (Ertel and Fowlkes 1976). Both methods of slope estimation have drawbacks: the spline fit often works well but requires some hand tuning; the chord-slope often underestimates the actual RLF slope. Nevertheless, the spline and chord slopes were correlated $\left(R^{2}\right.$ between 0.47 and 0.73 for different neuron types, $P<10^{-11}$ for all neuron types) and gave qualitatively similar results. The results of this paper do not depend on these slope estimates, which were done for comparison with previous work on the AN (Heinz and Young 2004); a problem with the slope estimates is that they are confounded by changes in maximum discharge rate and therefore difficult to interpret. The spline fits were used as a data reduction method based on which the RLFs could be classified into monotonic or non-monotonic. The following two features of the spline fit were used as the criteria for a non-monotonic shape: (1) firing rates below the $50 \%$ confidence interval of the spontaneous rate at levels above the threshold or (2) a decreasing slope located at levels above the threshold and spanning more than one eighth of the total level range (Cai 2007).

\section{Pseudopopulation analysis}

It is not possible to record large number of neurons in the cochlear nucleus with our methods, as can be done for example in constructing population responses in the AN (Pfeiffer and Kim 1975; Sachs 
and Young 1979). For this reason, we adopted the approach of taking as much data as possible from each neuron and studying fewer neurons. We were able to study an average of 10-12 VCN neurons per animal; in each neuron, data were taken at a number of different tone frequencies, as described above. To produce population estimates, we pooled neurons across multiple animals and used a pseudopopulation approach to study the RLFs. This method has been used previously in the CN (May et al. 1998). Frequency was expressed as the octave difference between BF and the tone frequency. Then, data were combined, ignoring the absolute $\mathrm{BF}$ and tone frequency and considering only the $\mathrm{BF}$ relative to the tone frequency. Figures 6, 7, 8, and 9 were constructed in this way, with the $\mathrm{BF}$ of the neuron expressed in octaves relative to the tone frequency. Each neuron usually contributed several RLFs to the average. For example, the data points at 1.2 octave on the abscissae of Figure 6 are averages of all the cases in which tone frequency was 1-1.4 octave below $\mathrm{BF}$ (e.g., a $2 \mathrm{kHz}$ tone presented to a $4.6-\mathrm{kHz}$ neuron or a $3.2-\mathrm{kHz}$ tone presented to a $7.4-\mathrm{kHz}$ neuron, etc.). Data were included only if both the $\mathrm{BF}$ and the stimulus frequency were below the CAP-audiogram edge frequency.

The pseudopopulation approach ignores the dependence of the properties of RLFs on BF. The assumption is supported by the fact that slopes of BF-tone RLFs did not vary significantly with BF in AN fibers in normal-hearing ears (Heinz and Young 2004). However, the thresholds of RLFs did vary systematically with BF. To correct for this effect, we defined an adjusted sound level scale dubbed dBP ("P" for "pseudopopulation") according to the following equation:

$$
L_{P}=L_{S P L}-\Theta_{\text {animal }}(B F)+\Theta_{\text {avg }}(2 k H z)
$$

where $L_{\mathrm{P}}$ and $L_{\mathrm{SPL}}$ are, respectively, the adjusted level and the original SPL level; $\Theta_{\text {animal }}(\mathrm{BF})$ is the CAP threshold of the animal at the $\mathrm{BF}$ of the neuron (in $\mathrm{dB}$ SPL $)$; and $\Theta_{\text {avg }}(2 \mathrm{kHz})$ is the $2-\mathrm{kHz}$ threshold (also dB SPL) of the average CAP audiogram of the corresponding animal group (exposed or unexposed). This adjustment expresses sound level as $\mathrm{dB}$ relative to the CAP threshold in the same animal $\left[L_{\mathrm{SPL}}-\Theta_{\text {animal }}(\mathrm{BF})\right]$, corrected to the average CAP threshold at $2 \mathrm{kHz}$. The subtraction of CAP threshold at $\mathrm{BF}$ eliminates systematic threshold variation with $\mathrm{BF}$ and AT and is analogous to expressing threshold in terms of SL, i.e., dB re threshold. Adding the average $2-\mathrm{kHz}$ threshold in normal or exposed animals restores the average threshold shift and expresses $L_{\mathrm{P}}$ in terms comparable to absolute sound level; this correction allows meaningful binaural balance calculations to be done, described below.
Weighted averages of the RLFs were calculated both within a PSTH type and then across different PSTH types. Because the relative numbers of different response types studied in this paper may not be representative of the relative sizes of the actual populations of VCN neurons, the averages were corrected using estimates of the relative fractions of different response types from previous studies, based on counts of anatomical cell types and counts of PSTH response types (Osen 1970; Bourk 1976; Cant and Morest 1984; Blackburn and Sachs 1989; Melcher 1993; Doucet and Ryugo 2006). The relative counts are as follows: Pri/PriN/ChS/ChT/ChL/On/Lock$\mathrm{er} /$ Unusual $=1 / 0.21 / 0.39 / 0.59 / 0.097 / 0.12 / 0.53 / 0.36$ (Cai 2007); that is, for example, there should be $21 \%$ as many PriN as Pri neurons and $12 \%$ as many onset neurons as Pri neurons. For the data shown in this paper, these weights were applied when averaging the Pri and PriN subpopulations to construct the overall primary-like (PL) group and to the $\mathrm{ChS}, \mathrm{ChT}$, and ChL subpopulations to construct the overall chopper group. All the categories were used in computing the estimates in Figure 6. The same sets of weights were used in the normal-hearing and exposed groups.

To estimate the uncertainty of average RLFs and rate-matching slopes, we performed bootstrapping on the datasets. For the pseudopopulation datasets, the bootstrapping procedures were based on repeated resampling of neurons in different hearing states and PSTH types. Five-hundred-time bootstrapping was done for each case.

Statistical comparisons in this paper are based on two-tailed Wilcoxon rank-sum tests on a significance level of 0.05 , unless stated otherwise.

\section{RESULTS}

\section{CAP audiograms and edge frequencies}

Data were obtained from 12 noise-exposed and nine normal-hearing cats, whose CAP audiograms are shown in Figure 1A. CAP audiograms were used in this study in lieu of recordings from AN fibers to characterize the degree of damage to the cochlea. For both the individual-experiment and the populationaverage audiograms, threshold shifts were greatest within two octaves of $2 \mathrm{kHz}$ (the shaded bar in Fig. 1A), near the frequency of the exposure noise. At lower and higher frequencies, threshold shifts generally decreased.

Although the extent and frequency span of threshold shifts varied between individual exposed animals, a sharply down-sloping portion at the high end of the threshold-elevated frequency region was invariably present, based on which the boundaries (edge frequencies) of the threshold-elevation could be 
defined, as described in "Materials and methods." The edge frequencies of the exposed animals ranged from 4.2 to $17.3 \mathrm{kHz}$, with a geometric mean of $11.3 \mathrm{kHz}$, which was arbitrarily chosen as the edge frequency of all the normal-hearing animals. In Figure 1B, the CAP audiograms are aligned at their edge frequencies, with frequencies expressed on a virtual frequency axis as octaves re the edge. It can be seen that the audiograms align reasonably well at the edge frequencies and that the virtual frequency axis provides an effective way to distinguish frequency ranges with and without consistent threshold elevations. Subsequent analysis of RLFs will be based on neurons with BFs below the edge. The amount of threshold shift below the edge frequency varied over about a 20-dB range. To compensate for this variation, sound level was expressed as $\mathrm{dB}$ relative to threshold in various ways, described below.

\section{Classification and quantification of PSTHs}

The major PSTH types of VCN neurons in normalhearing cats were found in noise-exposed animals as well. Most VCN neurons in noise-exposed animals could be readily classified into Pri, PriN, Chopper, Onset, Locker, and Unusual types according to the decision tree given by Blackburn and Sachs (1989). Table 1 summarizes the numbers of different types of VCN neurons recorded from normal and exposed animals, as well as the distributions of their BFs relative to the edge frequencies. No PSTH types seen in the normal animals were missing in exposed animals nor was there any evidence for well-defined abnormal PSTH categories in exposed ears. The "unusual" response type did not become more common, for example $\left(\chi^{2}=0.08\right.$, N.S. $)$. The distribution of response types was similar in normal and exposed animals, except that there were relatively fewer onset neurons and more Pri neurons at frequencies below the edge in exposed animals $\left(\chi^{2}=\right.$ 12.2, $P=0.002,2 d f)$.

The qualitative similarity of PSTH patterns in normal and exposed animals begs the question of whether AT altered any quantitative aspects of the responses. A consistently observed difference in normal VCN is first-spike latency, which is $\sim 1 \mathrm{~ms}$ shorter in primary-like neurons than in chopper neurons (Young et al. 1988; Blackburn and Sachs 1989); this difference was also observed in this study after AT $\left(P=4 \times 10^{-5}\right)$. In addition, we compared the sustained discharge rate, the coefficient of variation of interspike intervals, the standard deviation of firstspike latencies, and the rate of change of first-spike latency with level. At the levels tested, none of these measures differed significantly between the normal and exposed groups (not shown), except that the maximum sustained discharge rate was significantly higher in chopper neurons; changes in discharge rate are considered in "Tone rate-level functions," "Spread of excitation," and "Rate-matching curves."

The BF thresholds of neurons in normal and exposed ears are shown in Figure 2, in the form of threshold relative to the average normal CAP threshold at the neuron's BF (Fig. 1A), plotted against frequency relative to the edge frequency. Neurons of different response types showed similar threshold shifts, as expected if the threshold shifts were of cochlear origin. The single-neuron thresholds in normal animals (unfilled symbols) were on average below the CAP threshold because a suprathreshold response is necessary to achieve the CAP threshold criterion. In individual animals, the single neuron thresholds varied with $\mathrm{BF}$ in essentially the same way as the CAP thresholds (Cai 2007).

One hypothesis for recruitment is a compression in the range of neural thresholds following cochlear damage (Zeng and Turner 1991; Moore et al. 1985); that is, when thresholds are elevated, the distribution

TABLE 1

Numbers of neurons in different PSTH categories, counted separately for neurons below and above the edge frequency and for normal and exposed ears

\begin{tabular}{|c|c|c|c|c|}
\hline & \multicolumn{2}{|c|}{ Normal hearing } & \multicolumn{2}{|c|}{ Noise-exposed } \\
\hline & $B F \leq f_{E d g e}$ & $B F>f_{\text {edge }}$ & $B F \leq f_{\text {edge }}$ & $B F>f_{\text {edg }}$ \\
\hline Pri & 4 & 1 & 21 & 7 \\
\hline PriN & 7 & 5 & 13 & 6 \\
\hline Chopper & 12 & 6 & 16 & 13 \\
\hline Onset & 9 & 2 & 3 & 7 \\
\hline Locker & 5 & 0 & 11 & 0 \\
\hline Unusual & 9 & 2 & 12 & 4 \\
\hline Total & 46 & 16 & 76 & 37 \\
\hline
\end{tabular}

Not included are neurons for which there is insufficient data to allow classification $(18,10,23$, and 9 neurons, respectively across the table). Another 15 recorded neurons were judged to be of DCN origin and are not included 


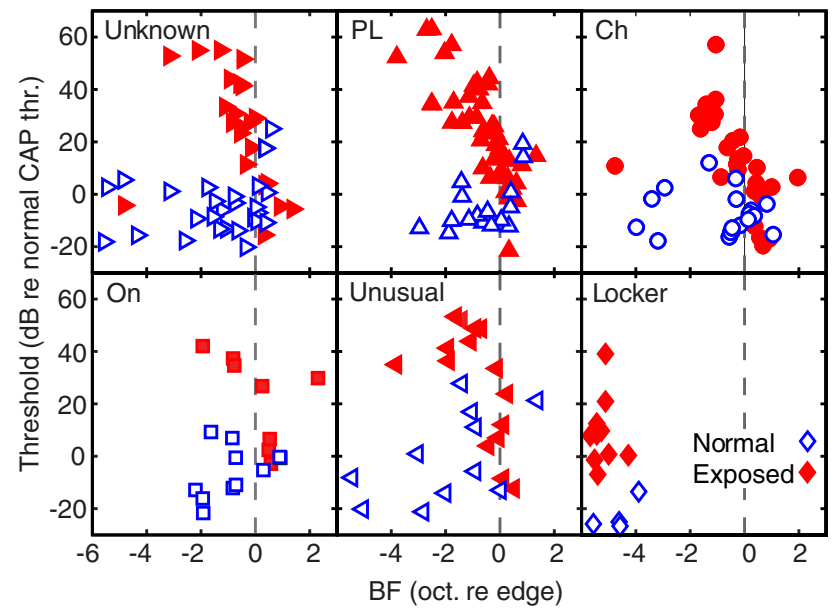

FIG. 2. Thresholds at BF of neurons of various response types plotted against BF relative to the edge of the CAP audiogram. The thresholds are presented $\mathrm{as} d \mathrm{~B}$ relative to the thresholds in the average CAP audiogram of the normal group (Fig. 1). Symbols identify neuron types; these are the same as in subsequent figures. Filled symbols are from exposed animals, and unfilled symbols are from normal animals.

of neural thresholds is compacted by the reduction in the possible range of threshold values, thus reducing the range of sound levels over which the summed rate of a population of auditory neurons can change, leading to recruitment. This hypothesis was not supported by AN data (Heinz et al. 2005); in the present data set, the range of thresholds was estimated as the standard deviation of neural thresholds relative to the CAP threshold at the neuron's BF. Locker neurons were not included in this calculation because of their low BFs but do not change the result if included. The resulting standard deviations are $9.5 \mathrm{~dB}(N=50)$ in normal animals and $11.4 \mathrm{~dB}(N=71)$ in exposed animals. Thus, the hypothesis of threshold range compression is not supported by these cochlear nucleus data.

\section{Sharpness of tuning}

In normal-hearing ears, the tuning curves of VCN neurons were similar to those of previously described AN fibers in terms of shape, threshold, and tuning quality. Figure 3A shows the 10-dB quality factors (Q10s) of normal (unfilled symbols) and exposed (filled symbols) VCN neurons. Most neurons from normal ears fell into the $95 \%$ confidence interval of Q10s of normative AN populations from Miller et al. (1997) and Ji (2000) (dashed lines), but many neurons from exposed ears had Q10s below the normal range.

To show the relationship of Q10 and threshold, we defined relative $Q 10$ as Q10 divided by the moving
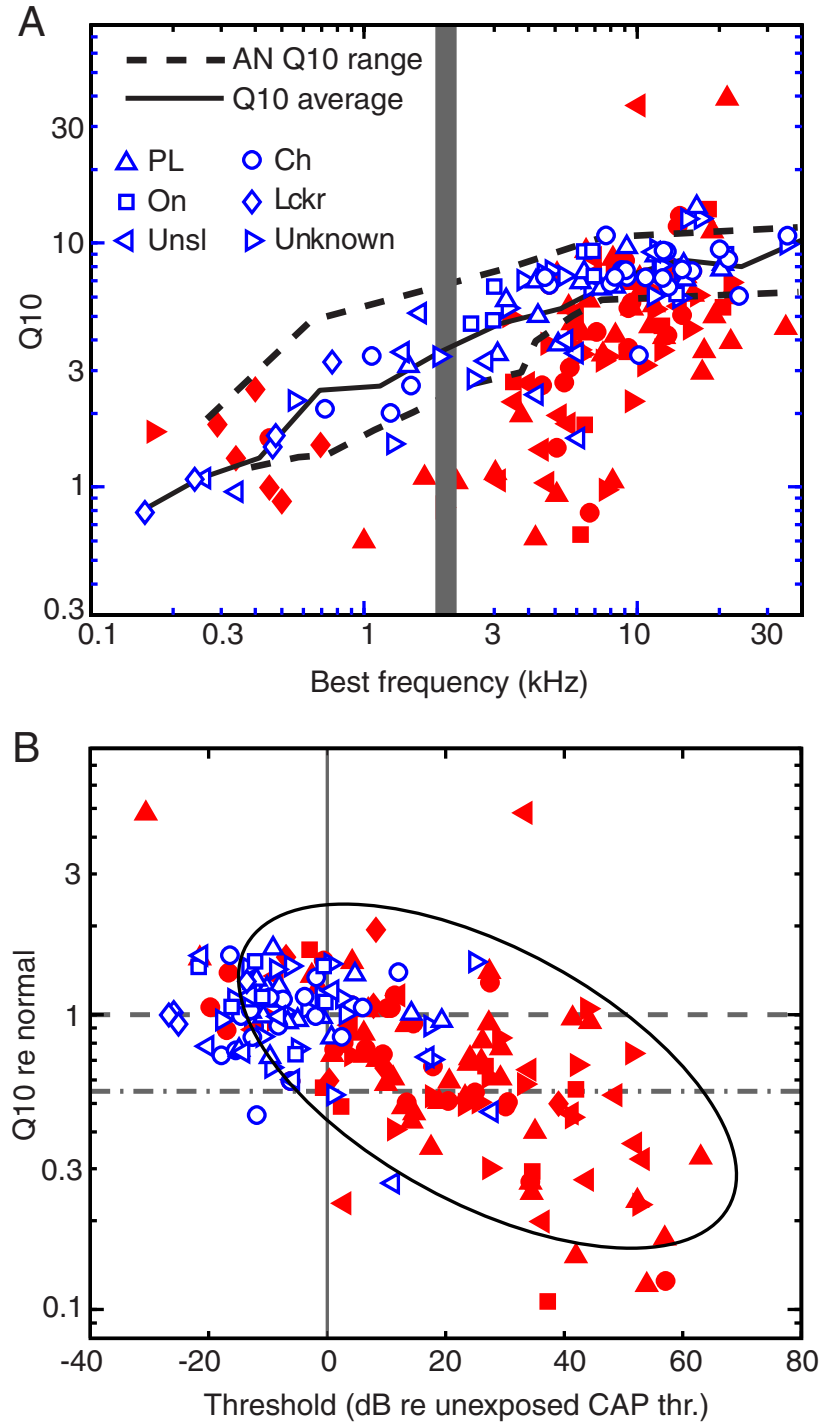

FIG. 3. Q10 values of $\mathrm{VCN}$ neurons are similar to those of $\mathrm{AN}$ fibers in both normal and noise-exposed ears. A A summary of tuning-curve Q10s of neurons in the normal (unfilled symbols) and noise-exposed (filled symbols) populations. The gray bar indicates the frequency of the exposure noise. The upper and lower limits $(5 \%$ and $95 \%$ quantiles) of Q10s of normal AN fibers from Miller et al. (1997) and Ji (2000) are overlaid for comparison (dashed lines). The solid line shows the moving average of Q10 versus BF for the control group. B Relative Q10 (normalized by the average Q10 at the same $\mathrm{BF}$ in the normal-hearing population) versus threshold relative to the average normal CAP audiogram (Fig. 1). The horizontal dashed line indicates the average normal Q10 values. The horizontal dash-dotted line (at 0.55 ) is the boundary between sharp and broad tuning used by Heinz and Young (2004), chosen to be the fifth percentile of Q10s in normal AN fibers. The ellipse shows the region occupied by AN fibers with moderate to severe loss from Figure 5 of Heinz and Young 2004 for comparison. The ellipse was shifted along the abscissa by $28 \mathrm{~dB}$ to account for the difference between the mean CAP threshold at $2 \mathrm{kHz}(23.5 \mathrm{~dB} \mathrm{SPL})$ and the threshold reference used by Heinz and Young at $2 \mathrm{kHz}(-4.5 \mathrm{~dB} \mathrm{SPL})$. 
average of Q10s of normal VCN neurons at the same BF (the solid line in Fig. 3A). The relative Q10s are plotted against threshold shift (the threshold minus the average normal CAP threshold from Fig. 1A) in Figure 3B. In the exposed neurons, there was a general trend for neurons with greater threshold shifts to have lower Q10 values $\left(P=5 \times 10^{-6}\right.$, first-order linear regression). The tuning exhibited little dependence on PSTH type. For comparison, the ellipse shows the region occupied by the AN fibers with moderate to severe hearing loss in a similar plot in Heinz and Young (2004). The overlap in the AN and VCN data suggests that the cochlear damage is similar in the two sets of exposed animals. Given this consistency, we will compare post-trauma alterations in RLFs between our VCN neurons and the AN fibers of Heinz and Young (2004).

\section{Tone rate-level functions}

Tone rate-level functions were recorded in 1-dB steps at multiple frequencies including BF. Figure 4A shows the driven rates, meaning total rate minus $\mathrm{SR}$, versus sound level for tones at $\mathrm{BF}$ in the Pri and PriN populations. Normal and exposed populations are indicated by the dashed and solid lines, respectively. These data are from neurons with BFs below the CAP edge frequency, so the neurons from exposed ears all had substantial threshold shifts. As can be seen in Figure 4A, PL neurons showed a mixture of RLF shapes. The majority showed a monotonic rate increase with level over the first 20-30 dB above threshold, followed by a decrease in slope or saturation. Of the normal PL neurons $45.5 \%$, predominantly PriN, showed non-monotonic RLFs; there was no significant difference in the number of PL neurons with nonmonotonic RLFs in the exposed and unexposed animals $(P=0.4$, Fisher's exact test $)$.

Visual inspection of Figure 4A suggests that threshold elevation was accompanied by diminished driven firing rates in PL neurons. To investigate this question, Figure 4B shows average RLFs for the normal and exposed PL population; the RLFs were aligned on threshold before averaging. Both average RLFs show a sigmoid shape with a dynamic range of approximately $20 \mathrm{~dB}$ but different rates of saturation. The mean saturated rates at $30 \mathrm{~dB}$ above threshold are 168 and 102 spikes/s in the normal and exposed PL populations, shown by the circles and error bars in Figure 4B (SEMs; $P=0.08$ ). The slopes of the BF RLFs of individual fibers were significantly different, slightly steeper for normal neurons (mean $\pm 1 \mathrm{SD}$ is $7.9 \pm 4.4$ spikes/s for normal and $4.7 \pm 2.7$ spikes/s for exposed neurons, $P=0.03$ ). Slopes at frequencies away from BF were not significantly different ( $P=0.2$, not shown).
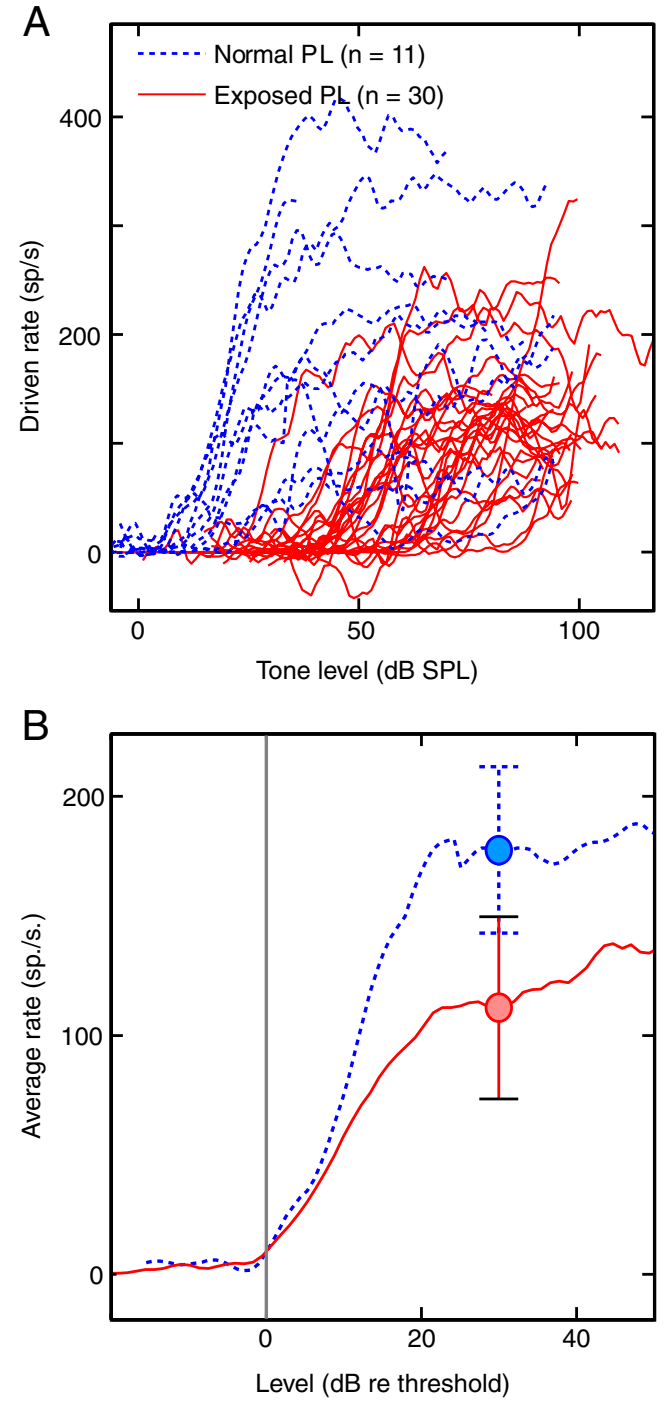

FIG. 4. Primary-like neurons show a decrease in rate responses following AT. RLFs from Pri and PriN neurons are shown. Data in normal and exposed animals are identified by line style, defined in the caption. A BF-tone RLFs as driven rate (rate-SR). B Average firing rate versus level re threshold. The individual RLFs in $\mathbf{A}$ were aligned at their thresholds (vertical line) and averaged. The circles and error bars show mean $\pm 1 \mathrm{SE}$ of rates at $30 \mathrm{~dB}$ re threshold.

The same measures applied to the chopper responses are shown in Figure 5. The chopper neurons showed a substantial increase in saturation driven rate in exposed animals. In the averaged rate functions (Fig. 5B), the saturation rates are 357 spikes/s in the exposed versus 234 spikes/s in unexposed animals $(P=0.02)$. In addition, the fraction of non-monotonic BF-tone RLFs was significantly smaller in the exposed animals $(28.6 \%)$ than in the unexposed animals $(83.3 \% ; P=0.007$, Fisher's exact test). The slopes of BF RLFs were significantly larger in exposed animals $(10.1 \pm 3.9$ in normals versus $14.9 \pm$ 4.8 spikes $/ \mathrm{s}$ in exposed ears, $P=0.02$ ), but the slopes at 

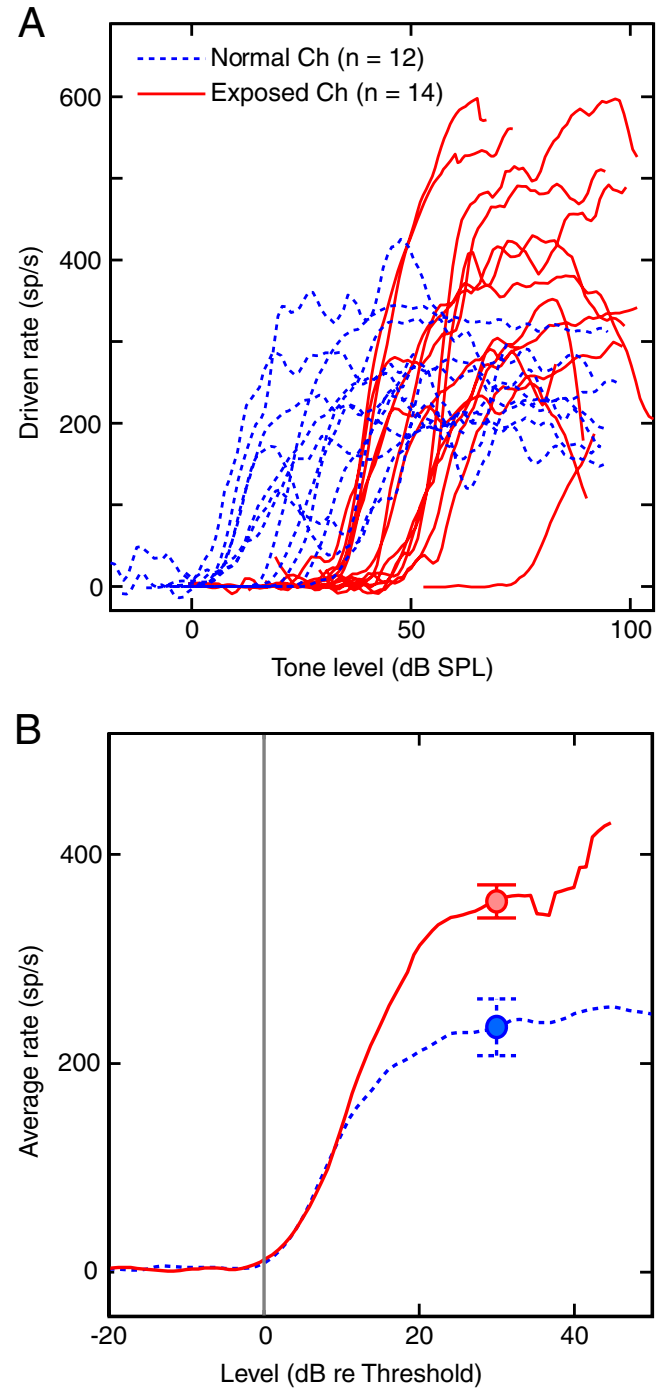

FIG. 5. Chopper neurons show an increase in rate response after AT. RLFs for BF tones in chopper neurons arranged as in Figure 4.

frequencies away from BF did not differ significantly $(P=0.1)$.

The results from other PSTH types are not presented separately. However, they are generally consistent with the results shown in Figure 5 (Cai 2007) in that onset and unusual neurons showed BFtone RLFs that were, on average, steeper than normal following sound exposure and saturated at higher rates. The low-BF Locker neurons showed a bimodal distributions of slopes, which presumably reflects a mixture of PL and non-PL neurons in this category.

\section{Spread of excitation}

Spread of excitation in the neural population is often considered to be an important parameter in the growth of loudness. Abnormally fast spread of excitation due to broadened tuning in impaired ears is one possible correlate of recruitment (Kiang et al. 1970; Evans 1975). Although spread of excitation in the AN array does not seem to be an important effect (Heinz et al. 2005), the properties of spread of excitation could be different in central populations, so estimates of the spread of excitation in VCN were derived here (Fig. 6). This analysis shows average normalized rate versus $\mathrm{BF}$ in octaves re the tone frequency. Rates were collected in 0.4 octave wide frequency bins, the approximate critical bandwidth in the cat (Pickles 1979). Because it is the activation of neurons that is
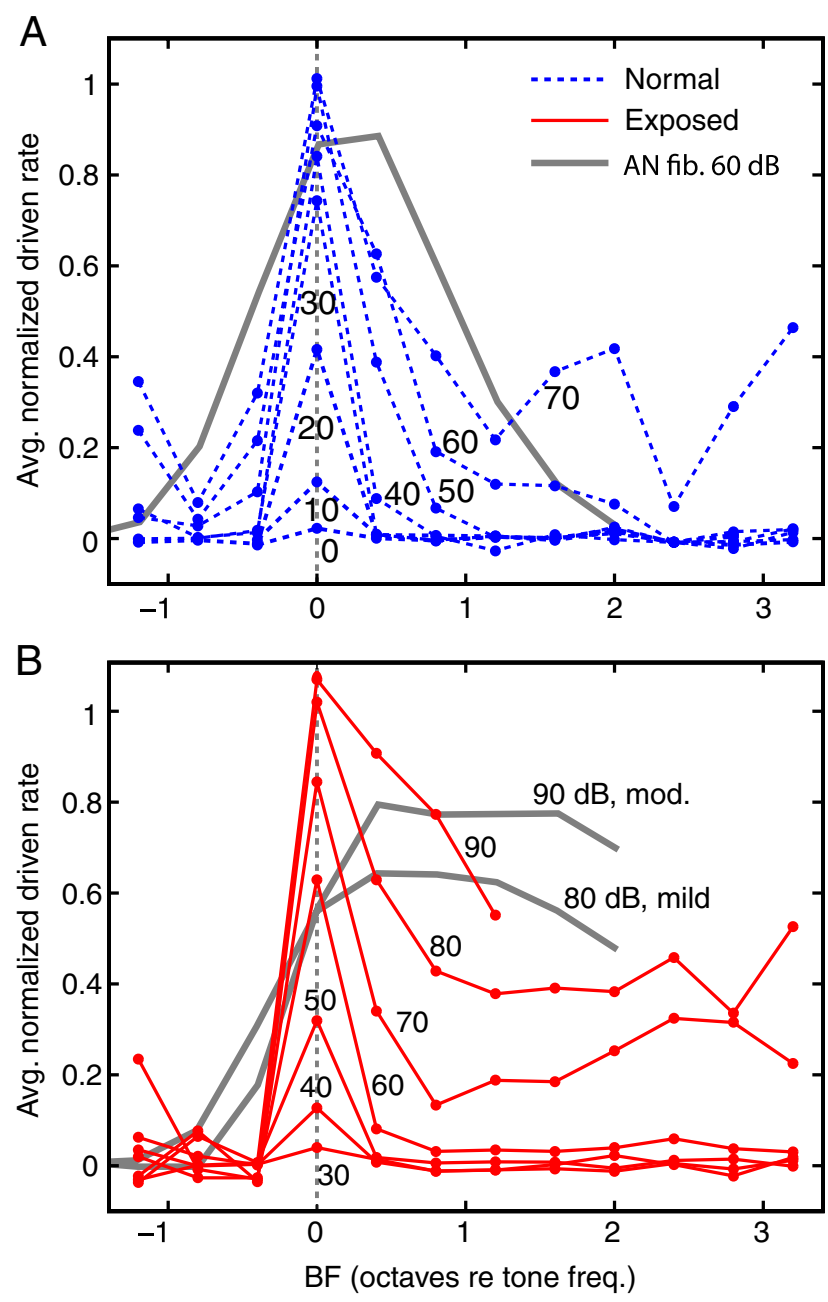

FIG. 6. Spread of excitation is larger following AT. A Equal-level rate contours for the tonal pseudopopulation in normal animals. The rates are average normalized driven firing rates of all VCN response types, computed in 0.4 octave frequency bins, as described in the text. The numbers show sound levels in $\mathrm{dBP}$. The heavy gray line shows the $60 \mathrm{~dB}$ SPL contour for AN fibers from Heinz et al. (2005), Figure 4, normalized in the same way. This level was chosen for comparison because it shows a clear shift in the BF at which the peak rate occurs. B Same for the exposed preparations. The heavy gray lines show 80 and $90 \mathrm{~dB}$ contours for AN fibers in preparations with "mild" and "moderate" hearing loss, as defined in Heinz et al. (2005). Locker neurons are not included in this figure because their BFs are far from those of other neuron types. 
important in spread of excitation, and not the overall discharge rate, the firing rates were normalized for each response type by the average driven rate in unexposed ears for a $\mathrm{BF}$ tone at $30 \mathrm{~dB}$ re threshold (e.g., the circles in Figs. 4B and 5B) and then combined across neuron types using the weights described in "Materials and methods." This prevents response types with high discharge rates (e.g., choppers in exposed ears) from dominating the plot. Thus, Figure 6 shows the average level of activation across PSTH types and not the average or overall rate in the pseudopopulation.

As expected, the degree of activation spreads as sound intensity increases. Comparing the normal (Fig. 6A) and exposed (Fig. 6B) populations, the difference in threshold is evident. There is also a difference in spread of activation in that the whole population was activated at $60-70 \mathrm{dBP}$ in the exposed population, about 20-30 dB above threshold, whereas activation did not spread across the whole population until $60 \mathrm{dBP}(>50 \mathrm{~dB}$ re threshold) in the normal population, which implies that spread of excitation was two to three times faster in the exposed ear than in the unexposed one.

Comparison AN data at moderate to high levels are shown by the heavy gray lines (from Fig. 4 of Heinz et al. 2005). Note that the broadening of the active population is not as prominent in VCN as in the AN, especially in the exposed population (Fig. 6B), and the shift in the peak of the curves toward BFs above the tone frequency is smaller or non-existent in the VCN, consistent with iso-level rate plots in previous studies (Rhode and Smith 1986; Rhode and Greenberg 1994). In this study, a shift in the response peak did not occur up to $70 \mathrm{dBP}$ in the normal population (Fig. 6A), whereas in the AN fibers, it was seen at levels of 50-60 dB SPL in both normal and impaired populations. These levels correspond to $\sim 50 \mathrm{~dB}$ above threshold in the normal AN fibers and 20-30 dB above threshold in fibers following AT. Thus, the peak of the rate plots is more stable in VCN than AN.

\section{Rate-matching curves}

The summed firing rate of a population of auditory neurons is usually considered to be the neural correlate of perceptual loudness, although how summed rates translate to loudness is not clear (Pickles 1983; Relkin and Doucet 1997; Heinz et al. 2005). To compare the behaviors of VCN population rates and loudness, we estimated population firing rates using the pseudopopulation method described in "Materials and methods." Evidence has been offered that the population involved in encoding the level of a tone may not be the entire $\mathrm{BF}$ range, but only BFs near the tone frequency (Hellman 1978; Moore et al. 1985; reviewed by Smith 1988), so the population firing rate was estimated for several $\mathrm{BF}$ ranges and also separately for the VCN neuron types.

In psychophysical studies of loudness recruitment, a frequently used paradigm is loudness balance between the two ears. In this paradigm, the loudness in one ear with a near-normal threshold is adjusted (by varying sound level) to match the loudness in an impaired ear across a range of sound levels. The slope of the generated loudness matching curve quantifies the rapidity of loudness growth in the impaired ear relative to normal (Miskolczy-Fodor 1960; Stillman et al. 1993; Moore et al. 1985; Zeng and Turner 1991; Moore 2004). In this study, loudness balance was simulated by matching discharge rates in normal and exposed pseudopopulations. The calculations were done by interpolating in the two RLFs to find the sound levels that produce a series of rates between threshold and saturation (or the first turning point in either RLF).

Rate-matching curves for on-BF and all-BF encoding in three different populations are shown in Figure 7. In this figure, "on-BF" means that the BF was within \pm 0.2 octaves of the tone frequency and "allBF" means that RLFs were included over all frequencies, as long as both tone frequency and BF were below the edge frequency. The left panel of each part of Figure 7 shows average RLFs for the normal (dashed lines) and noise-exposed (solid lines) populations. Bootstrapping was used to estimate the standard deviations of the rate estimates; \pm 1 SD confidence limits are shown as shaded regions for the on-BF pseudopopulations. The all-BF pseudopopulation results are shown without confidence limits, but the limits are similar to the ones shown for the on$\mathrm{BF}$ data (the presence of shading also serves to separate the on-BF from the all-BF data). The ratematching curves for on-BF and all-BF encoding are shown in the right panels, identified by line color, as in the legend. For comparison, the dashed line in the right panels shows a typical rate-matching curve based on the reported loudness-balance data in human observers (Miskolczy-Fodor 1960; Stillman et al. 1993; Moore et al. 1985; Zeng and Turner 1991; Moore 2004). This is a straight line that extends from a point defined by the thresholds in the normal and impaired ears to the point $(100 \mathrm{~dB}, 100 \mathrm{~dB})$, assuming that loudness is the same in normal and impaired ears at $100 \mathrm{~dB}$. Linear regression was performed on the rate-matching curves to give their overall slopes, which are shown next to the lines. Note that $100 \mathrm{~dB}$ is a high level for the equal-loudness level; however, choosing a lower level would increase the slope of the dashed line, effectively increasing the slope that neural rate balance functions have to achieve to be considered consistent with recruitment. For the 


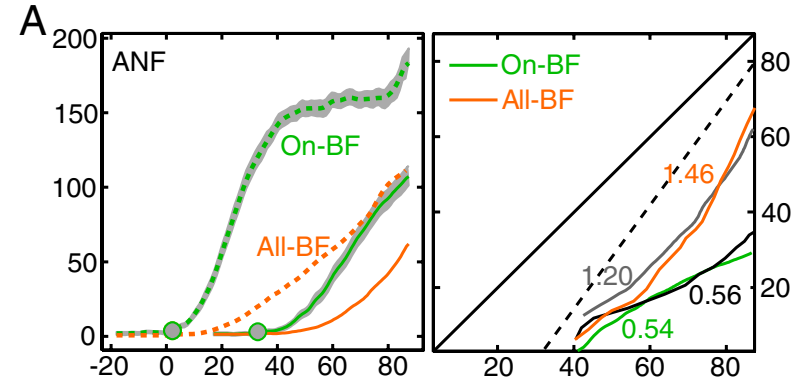

$\mathrm{B}$
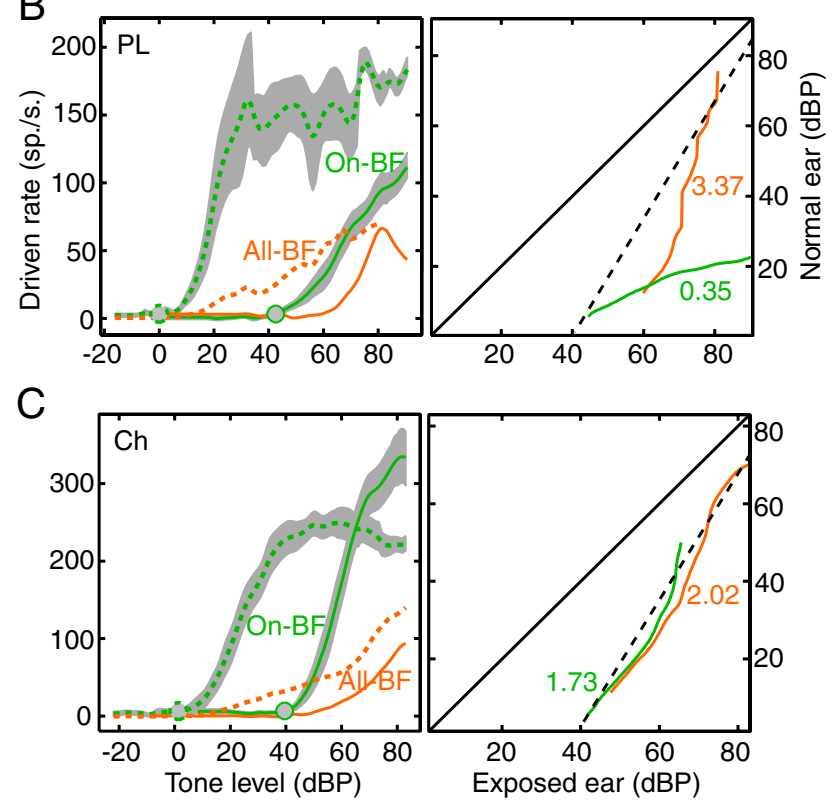

FIG. 7. Rate-balance functions from $\mathrm{Ch}$, but not $\mathrm{PL}$ neurons resemble perceptual loudness-balance functions. In each section, the left plot shows the average rate for normal (dashed lines) and exposed (solid lines) pseudopopulations consisting of (1) neurons with BFs within \pm 0.2 octaves of the tone frequency $(O n-B F)$, shown with shaded bootstrap confidence limits $( \pm 1 \mathrm{SD})$, and (2) neurons of all BFs, shown as the average rate lines only. The circles on the on-BF functions show the thresholds (3 spikes/s). The right plots show the sound levels that give the same rates in the normal and exposed populations. Matches are shown for the on-BF and all-BF populations, identified by line shading, as in the legend in $\mathbf{A}$. The numbers are the slopes of best-fitting lines for the rate matches over the range of sound levels shown. The dashed lines show predictions for typical recruitment functions in human observers (see text). A AN data from Heinz et al. (2005), reanalyzed with the pseudopopulation method. Rate-matching lines and slopes are shown for both pseudopopulation analysis (slopes, 0.54 and 1.46) and from the previous realpopulation analysis done by Heinz and colleagues (slopes, 0.56 and 1.20). B, C Average RLFs and the corresponding rate-matching curves for PL and chopper neurons.

comparisons below, that change would make it easier to conclude that neural slopes are smaller than expected for recruitment and, in this sense, the choice of $100 \mathrm{~dB}$ is conservative.

Figure 7A shows a pseudopopulation response constructed from the AN data of Heinz et al. (2005).
RLFs with tone frequencies and neuron BFs between 0.5 and $6 \mathrm{kHz}$ (the most impaired $\mathrm{BF}$ region) from animals with moderate/severe SNHL were used. AN fibers were averaged within SR groups (low+medium, high) before combining those averages with weighting according to the expected numbers of fibers in each SR group from previous studies (0.4 and 0.6 respectively; Liberman and Dodds 1984). The resulting on-BF RLF has a smaller slope in impaired ears compared to normal, giving a rate-balance line with slope 0.54 . The all-BF RLF for the impaired pseudopopulation has an accelerating slope that is smaller than normal at low levels but increases rapidly at high levels. The rate-matching function has a slope less than the recruitment line at sound levels within $20 \mathrm{~dB}$ of threshold, but the slope increases at higher levels, giving an overall slope of 1.46 . For comparison, the rate-matching curves from the earlier real-population calculation of Heinz and colleagues are superimposed in the right-hand plot (slopes, 0.56 and 1.20). The two sets of rate-matching curves are essentially identical, suggesting that the pseudopopulation method gives accurate results for the rate-matching curves.

The behavior of the PL pseudopopulation (Fig. 7B) is similar to that of AN fibers, except that the standard deviations of the RLFs are larger. The rate matching slope $(0.35)$ is again considerably smaller than the expected recruitment behavior in the on-BF population. The all-BF RLF accelerates considerably at high sound levels, giving a steep ratematching function (slope, 3.37) beginning about $20 \mathrm{~dB}$ above threshold.

The all-BF rate matching function of the PL neurons, and to a lesser degree also the AN fibers, seems at first glance to be consistent with recruitment, in that steep slopes were obtained over most of the range of sound levels. However, in AN fibers, there is a low-slope segment near threshold, over the first $20 \mathrm{~dB}$ in the impaired ear. This low-slope portion must also be present in the PL neurons (Fig. 7B) because the on-BF rate-matching curve of the PL neurons has a shallow slope. But the low-slope portion in the all-BF curve is not plotted because the slow growth of the rate balance function over the first $10 \mathrm{~dB}$ above threshold was obscured by noise in the all-BF RLF. However, the rate-matching curve must begin at the point defining threshold in the two ears, $42 \mathrm{~dB}$ and $0 \mathrm{~dB}$ for the PL neurons (Moore 2004), meaning that the PL neurons' rate-matching curve must include a low-slope portion similar to the on-BF rate matching curve.

The chopper neurons (Fig. 7C) showed summed RLFs, which were quite different from those of the AN fibers and PL neurons, in that the impaired population showed a steeper on-BF RLF than its normal counterpart. This steepened slope, together with the 
absence of rate saturation, caused the on-BF ratematching curve to have a steep slope beginning at threshold (1.73), consistent with loudness growth in recruitment. The all-BF average rates are smaller than the on-BF ones but again have a steeper slope, giving a rate matching curve that also matches expected recruitment behavior (slope, 2.02).

Spread of excitation changes rate-matching for primary-like but not chopper neurons

On-BF and all-BF are the two extremes of the possible $\mathrm{BF}$ ranges of neurons participating in intensity encoding. Figure 8 shows how the slopes of the ratematch functions vary with the frequency window width of the analysis. The rate-matching computation was done for window widths from 0.4 to 6.8 octaves in 0.4-octave steps for the pseudopopulations analyzed in Figure 7. The BF window was always centered at the tone frequency. As in Figure 7, the slopes are often a compromise between shallow values near threshold and steep values at higher levels. Bootstrapping was performed for each pseudopopulation and each window width to generate estimates of uncertainty, given as $\pm 1 \mathrm{SD}$ error bars in Figure 8 . The shaded

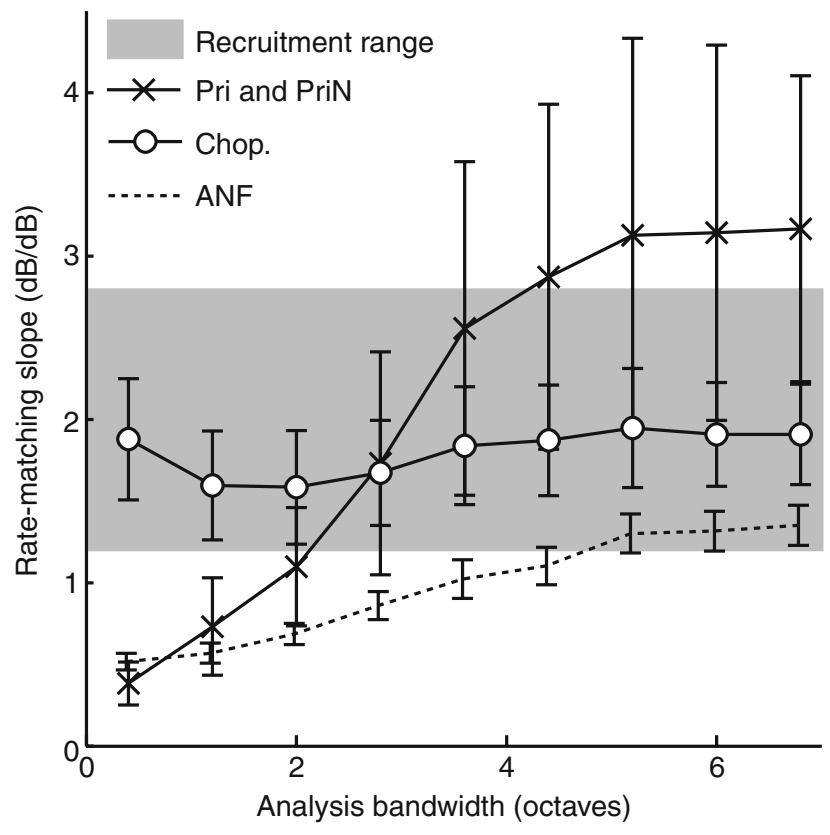

FIG. 8. The slopes of rate-matching functions change with the averaging bandwidth for $\mathrm{AN}$ fibers and PL neurons, but not $\mathrm{Ch}$ neurons. Slopes of rate-matching functions are shown for tonal pseudopopulations as a function of the range of BFs (relative to the tone frequency) averaged together. Results are given as mean \pm 1 SD of 500 bootstrap repetitions of the calculation for the same three populations as in Figure 7, identified in the legends. The shaded band shows the range of slopes of loudness-matching curves in human subjects (publications cited in the text) with hearing losses comparable to those of the exposed cats. The AN fiber data are from a reanalysis using pseudopopulations of the data of Heinz et al. (2005). region shows the range of loudness-balance slopes from several psychophysical studies (Miskolczy-Fodor 1960; Stillman et al. 1993; Moore et al. 1985; Zeng and Turner 1991; Moore 2004). Cases with threshold shifts between 30 and $50 \mathrm{~dB}$ were chosen, and the median (1.9) and interdecile range (1.2-2.8) of the slopes are plotted as the gray region. There is a tendency for the rate-matching slopes to increase from values less than the psychophysical data at narrow window widths to values consistent with or larger than the psychophysical values at wide window widths. The exception is the choppers that show recruitment-like slopes for all bandwidths. AN fibers have the lowest slopes across the range of bandwidths and barely reach the recruitment region for all-BF encoding.

Rate-matching curves for BBN show the same effects as for tones

Figure 9 shows the average RLFs and rate matching curves for responses to BBN. For noise, there is no meaningful definition of on-BF, so results are shown for the all-BF pseudopopulations only. In the left part of the figure, the average RLFs for primary-like and non-primary-like (Ch, On, and Unusual) VCN neurons are compared. The different PSTH types were weighted as in Figure 6 when averaging, but the rates were not normalized. The rate-matching curve of the PL neurons in the plot at right showed a shallow slope (0.60) similar to that of the AN fibers and PL neurons in Figure 7, whereas the non-PL neurons showed a steeper rate-matching slope (2.77), like the $\mathrm{Ch}$ neurons in Figure 7 . This shows a clear recruitment-like effect in the non-primary-like neurons in VCN.

\section{DISCUSSION}

\section{VCN response properties after acoustic trauma}

Following AT, neurons in the VCN showed threshold shifts and broadened tuning over a wide range of frequencies centered on the exposure frequency (Figs. 2 and 3). The changes were qualitatively and quantitatively similar to those seen previously in $\mathrm{AN}$ fibers following similar exposures (Miller et al. 1997; Heinz and Young 2004). Moreover the VCN threshold shifts were consistent with the CAP thresholds in the same animals in that VCN neural and CAP thresholds varied similarly with frequency (Cai 2007). Beyond the threshold and tuning changes, the main effects of AT on VCN neurons were changes in the RLFs. It is important that there was no evidence for significantly altered PSTH properties in VCN neurons. The usual VCN response types were observed after AT, and their properties, such as regularity and latency, were not 


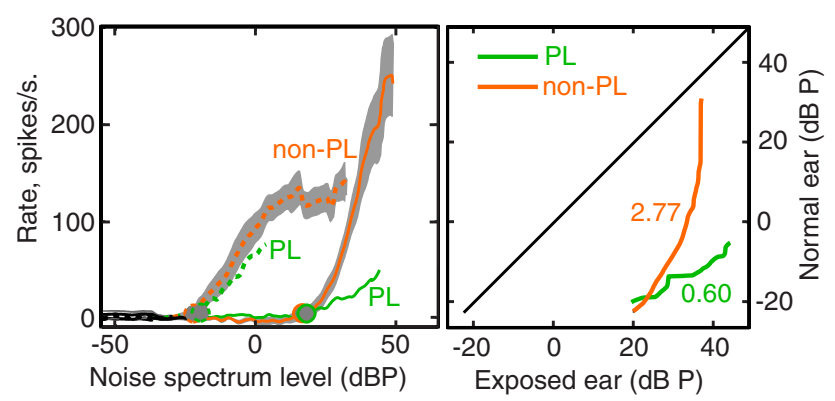

FIG. 9. Rate-matching slopes for responses to BBN; these differ between PL and non-PL neurons as for the tone responses. The figure is drawn as in Figure 7 , except that the recruitment prediction line is not shown because of uncertainty as to how recruitment should behave for BBN. Neurons are grouped into PL and non-PL pseudopopulations, and the plots show average RLFs (left) and ratematching curves (right).

significantly altered. This allowed the analyses done in this study to be based on PSTH response types established in normal animals. It also makes it possible to assume that the relationships between response types and morphological cell types established in normal animals also hold in impaired animals.

The observation that all major PSTH types were still present after AT is consistent with previous intracellular studies, which showed that basic membrane properties of VCN neurons in animals with cochlear damage or ablation are similar to normal (Francis and Manis 2000; Wang and Manis 2005; Lu et al. 2007). Although changes in a number of parameters of postsynaptic ion channels have been reported in the medial nucleus of the trapezoid body after AT (Leao et al. 2004, 2005, 2006), in the $\mathrm{CN}$ it is clear that the important differences between so-called types I and II neurons (Oertel 1983) remain following AT. Type I neurons are stellate cells that show long time constants and good temporal integration; they respond to steady current injection with a maintained spike train. Type I neurons seem to correspond to choppers in vivo. Type II neurons are bushy cells, which have short time constants and fire only a few spikes at the onset of a current step. The difference is largely due to the presence of low-threshold potassium channels in type II membranes (Manis and Marx 1991; Rothman and Manis 2003). They correspond to PL neurons in vivo.

\section{Rate functions of primary- and non-primary-like neurons after trauma}

One interesting result of this study is the difference in the behavior of RLFs between PL and non-PL (especially chopper) neurons. In PL neurons, rates in response to $\mathrm{BF}$ tones were smaller following trauma (Fig. 4A, B); by contrast, chopper rates were substantially larger (Fig. 5A, B). Lonsbury-Martin and Martin
(1981) previously showed that, in acutely deafened rhesus monkeys, a subpopulation of VCN neurons showed diminished rate-level responses, whereas another subpopulation showed steepened RLFs. However these neurons were not identified by response type.

PL responses are recorded from anatomical bushy cells, which are innervated by AN fibers through large secure synaptic endings (Ryugo 1992). At these synapses, postsynaptic potentials are large, and the time constants of the postsynaptic neurons are short, which prevents temporal summation of synaptic events (Oertel 1985; Raman and Trussell 1992; Isaacson and Walmsley 1996). Presumably, PL and AN RLFs were similar because the operating mode of these synapses is to produce a postsynaptic spike whenever there is a presynaptic spike, as demonstrated by their complex action potentials which contain both a pre- and a postsynaptic potential (Pfeiffer 1966; Bourk 1976; but see Kopp-Scheinpflug et al. 2002). Bushy-cell synapses are altered in morphology following cochlear damage (Ryugo et al. 1997; Redd et al. 2000), but the implications for synaptic transmission of these morphological changes are not clear. In fact, some evidence suggests that AN/VCN synapses are strengthened following cochlear degeneration (Oleskovich and Walmsley 2002). However, as long as the postsynaptic potentials produced in bushy cells by AN fibers are above threshold, bushy-cell RLFs should remain like those of AN fibers.

The discussion above applies strictly to spherical bushy cells. The nature of synaptic integration in globular bushy cells is not completely understood (e.g., Smith and Rhode 1987; Joris et al. 1994; Rothman and Young 1996; Young and Sachs 2008), but probably shares many features with spherical bushy cells because of similarities like irregularity of spike timing and strong phase locking. In this study, we did not see a difference in behavior between Pri and PriN neurons, suggesting that the effects of AT are the same in spherical and globular bushy cells. Overall, the present evidence suggests that bushy cells do not change their operating mode following AT, as shown in Figures 4 and $7 \mathrm{~B}$.

After AT, chopper neurons and some other nonprimary-like VCN neurons exhibited enhanced rate responses (Fig. 6). Given the reduced rate responses of AN fibers, this observation suggests that posttrauma neuronal plasticity strengthened the effects of AN synapses on these neurons. The morphological correlate in VCN of non-primary-like PSTH types is the stellate or multipolar cell plus the octopus cell (Rhode et al. 1983; Smith and Rhode 1989), although it is unlikely that many octopus cells are included, based on PSTHs. Normally, AN synapses on stellate cells produce subthreshold postsynaptic responses (Oertel 1985; Ferragamo et al. 1998; Golding et al. 
1995), which require temporal summation of several EPSPs to exceed threshold. Thus, the discharge rates of stellate cells are likely to be affected sensitively by changes in either the strength of AN synapses or the postsynaptic processing. AN synapses on stellate cells have been shown to undergo less severe morphological changes than bushy synapses in congenital deafness (Redd et al. 2002). Because the basic postsynaptic properties of these neurons seem not to change after cochlear damage (Francis and Manis 2000), they probably still rely on temporal summation for spiking after AT. The increased discharge rate could be produced by various effects including (1) an increase in postsynaptic resistance (Fancis and Manis 2000), (2) changes in postsynaptic excitability or the density of postsynaptic receptors, (3) a decrease in inhibitory inputs to the cells, (4) presynaptic changes that strengthen the synapse (Oleskevich and Walmsley 2002), or (5) the formation of new synaptic inputs from AN fibers or other VCN neurons, e.g., through axonal sprouting (Kim et al. 2004).

A variety of evidence from prior studies suggests that inhibitory synapses in auditory neurons are weaker following cochlear damage or ablation (Willott et al. 1997; Suneja et al. 1998; Milbrandt et al. 2000; Mossop et al. 2000; Vale and Sanes 2000; Vale et al. 2003, 2004). Moreover, inhibitory effects on spike trains have been shown to be weaker following AT in the inferior colliculus and dorsal cochlear nucleus (Salvi et al. 2000; Ma and Young 2006). Inhibitory responses were not directly studied in this work. However, the decreased prevalence of non-monotonic RLFs in choppers following AT is consistent with a loss of inhibitory effects on the cells.

\section{VCN effects of changes in spatiotemporal patterns in the AN}

The discussion above treats VCN responses as resulting from standard feedforward synaptic processing. However, in an important paper, Carney (1994) has proposed a model in which VCN neurons are sensitive through coincidence detection to the relative temporal patterns of AN spike times across BF. Because the timing of AN spikes varies with sound level, this monaural coincidence detection could be used to encode both intensity differences (Colburn et al. 2003) and loudness. Furthermore, it could offer a possible explanation for loudness recruitment (Carney 1994). In an earlier paper, Carney (1990) showed sensitivity to the relative timing of AN spikes across BF in VCN neurons, including PriN, Onset, and Chopper neurons. However, it is not clear how important this mechanism is for the results shown in this paper, for two reasons. First, for tone stimuli, the coincidence mechanism only applies to low frequencies (those with phase-locking), and most neurons in this paper had BFs above $3 \mathrm{kHz}$. Recruitment in SNHL patients is observed at high tone frequencies as well as low (e.g. Stillman et al. 1993). Moreover, Figure 9 shows that the $\mathrm{Ch}$ and PL slopes also change differently for BBN, for which coincidence works at all BFs, because of envelope fluctuations. Thus, there is no evidence in our data for a connection between the changes in RLFs and the availability of information in monaural coincidence detection. Second, Carney (1990) showed coincidence effects in both PriN and Ch neurons, so it is not clear why these two neuron types should behave differently after AT (as in Figs. 4 and 5).

\section{The pseudopopulation method}

In order to combine data across animals with varying degrees of acoustic trauma, the pseudopopulation method was developed. To prevent including responses from normal neurons, the pseudopopulation was limited to neurons with BFs below the edge or cutoff frequency of the cochlear damage as estimated by the CAP threshold shift; in addition, responses to particular tone frequencies were included only if the tone frequency was below the edge. To further compensate for the varying degrees of threshold shift within the damaged $\mathrm{BF}$ region, sound levels were expressed as $\mathrm{dBP}$ (Eq. 1), which essentially expresses levels as $\mathrm{dB}$ relative to the CAP threshold at the neuron's BF. For tone frequencies near the $\mathrm{BF}$ of a neuron (i.e., in the On-BF calculations), this method is appropriate, controlling between-animal and across-frequency variability. However, it does combine data from neurons with different degrees of threshold shift, which is unavoidable with the data base available in this paper. Given the changes in BF RLFs shown in Figures 4 and 5 , combining across neurons should cause the estimates of population RLFs to be a compromise between RLFs of varying severity, but should not affect the principal results reported in this study, especially the differences between primary-and non-primary-like neurons. For tone frequencies away from BF, there is the additional problem that the difference of thresholds between tuning-curve tips and tails varies with the degree of threshold shift (e.g., Liberman and Dodds 1984), which introduces an additional variable into the calculation. This problem mainly affects all-BF calculations; again, it is not clear that it should affect the results in this study in a qualitative way.

\section{Implications for loudness recruitment}

Generally loudness is assumed to be proportional to the summed activity of some group of auditory neurons (e.g., Moore and Glasberg 2004), but the nature of that summation and the relevant popula- 
tions of neurons are unclear (Pickles 1983; Relkin and Doucet 1997; Heinz et al. 2005). Similar questions remain in the coding of somatosensory perceptual intensity (Johnson 1974; Muniak et al. 2007). Attempts to reproduce perceptual loudness functions by summing the activities of populations of AN fibers suffer from the difficulty that it is not clear exactly how the degree of match of the loudness and neural growth functions should be evaluated, given that they are both monotonically increasing functions of sound level.

In considering recruitment, these difficulties can be bypassed to some extent by assuming that changes in the loudness function should be mirrored by similar changes in neural response growth functions, steeper neural growth functions corresponding to steeper loudness growth, for example. Previous studies based on evoked potentials and multi-neuron recording showed recruitment-like overexcitability of central auditory neurons following cochlear damage in a variety of animal models (e.g., Saunders et al. 1972; Popeláŕ et al. 1987; Salvi et al. 1990; Syka et al. 1994; Szczepaniak and Møller 1996; Wang et al. 2002). This behavior has also been reported in the human auditory system through functional imaging (Morita et al. 2003; Langers et al. 2007). These neural data match the expectation of recruitment qualitatively in that the neural responses of populations are stronger after cochlear damage. Neural hyperexcitability is commonly reported in cortex and inferior colliculus, but the results in cochlear nucleus have been mixed, with both positive (e.g., Saunders et al. 1972) and negative (e.g., Salvi et al. 1990; Qiu et al. 2000) results. The different directions of change reported in this paper in the RLFs of different types of VCN neurons provide one possible explanation for this disagreement. From our results, it is clear that evoked potential amplitudes in VCN would be sensitive to which neurons dominate the response for the particular stimuli and electrode placements used. Nonetheless, the current study unequivocally suggests that the VCN is the most peripheral stage of the auditory pathway at which overexcitability of neurons appears following AT (e.g., Fig. 5). Moreover, it points to the synaptic processing in the non-primary-like, especially the stellate, cells as the initial locus of central compensation for reduced rate responses in the AN.

A more quantitative comparison of perceptual and neural response growth can be obtained by comparing loudness balance functions to rate-equality functions, as was done in Figures 7, 8, and 9. Equating the perceptual loudness between a normal and a damaged ear should behave similarly to equating the summed discharge rates of corresponding groups of neurons as long as loudness is in some way a monotonic function of summed neural activity. This approach avoids the difficulties posed by nonlinearities in the loudness growth functions themselves (e.g., Allen 1997; Buus and Florentine 2002; Moore and Glasberg 2004).

The rate-balance functions in Figure 7 were compared to an assumed recruitment function (dashed lines) in which loudness grows linearly from a point at the threshold (zero loudness) in the two ears to loudness equality at $100 \mathrm{~dB}$. Real loudness balance functions deviate from this assumed line in two ways: (1) Loudness matches can show a slope near 1 for the first $10 \mathrm{~dB}$ or so above threshold and then become steeper (Moore 2004), and (2) loudness may not be equal in the two ears at $100 \mathrm{~dB}$ (Moore 2004; Zeng and Turner 1991; Stillman et al. 1993). Equality can occur at lower sound levels or loudness in the damaged ear may not reach equality with loudness in the good ear, say in cases with conductive hearing loss. Neither of these deviations can lower the loudnessbalance slopes to the values shown by the AN fibers and PL neurons.

Because of these uncertainties, we compared rateequality and loudness-balance functions by computing their slopes over a range of 20-40 dB near threshold. The unity slope in the first $10 \mathrm{~dB}$ was not separately considered and was usually not apparent in the data. These results, summarized in Figure 8, show that chopper neurons (and other non-PL neurons, not shown) behave in a way similar to human observers regardless of summation bandwidth. Other populations matched the psychophysical data only for wideband summations that include most of the population of active neurons.

Interpretation of the rate-balance results depends on the psychophysical evidence suggesting that recruitment for tones depends primarily on activity in the auditory filters near the tone frequency and not on spread of excitation (Hellman 1978; Moore et al. 1985; Zeng and Turner 1991). Figure 8 makes it clear that spread of excitation has a larger effect in the VCN than in the AN, in that spread of excitation causes substantially steeper rate-balance slopes in VCN than in AN. This conclusion is most clearly shown by comparing the AN and PL slopes in Figure 8. These results suggest that recruitment, and perhaps by extension loudness, depends on non-PL neurons in VCN, particularly the chopper neurons.

An additional aspect of chopper responses following $\mathrm{AT}$ is their elevated discharge rates at high sound levels (Fig. 5). This behavior could provide an explanation for auditory hyperacusis (e.g., Sherlock and Formby 2005; Nelson and Chen 2004) in which intense sounds become too loud for comfortable listening. Of course, this hypothesis depends on the nature of the additional adjustments in rate responses at later stages of the auditory system. Presumably, 
there are mechanisms that regulate the strengths of synapses between cochlear nucleus and inferior colliculus neurons to keep the collicular neurons within their dynamic ranges. Depending on those rules, the increase in rate response of chopper neurons could be accompanied by an increase or a decrease in synaptic strength at the next stage (as in the peripheral tinnitus model of Shaette and Kempter 2006), producing varying degrees of recruitment and hyperacusis. These questions require further research.

\section{ACKNOWLEDGMENTS}

We would like to thank Michael Heinz for providing us with raw data from the previous auditory-nerve study. Ben Letham helped in a few sound exposure and neural recording sessions. Paul Nelson, three anonymous reviewers, and Philip Joris made helpful comments on the manuscript. We are grateful to Phyllis Taylor, Ron Atkinson, and Qian Gao for technical assistance. This study was supported by NIH/NIDCD grant DC00109 and core grant DC005211.

\section{REFERENCES}

AlLen JB. Derecruitment by multiband compression. In: Jesteadt W (ed) Modeling Sensorineural Hearing Loss. London, Erlbaum, 1997.

Blackburn CC, Sachs MB. Classification of unit types in the anteroventral cochlear nucleus: PST historgrams and regularity analysis. J. Neurophysiol. 62:1303-1329, 1989.

Bourk TR. Electrical Responses of Neural Units in the Anteroventral Cochlear Nucleus of the Cat. Unpublished doctoral thesis. Department of Electrical Engineering and Computer Science, Massachusetts Institute of Technology, Cambridge, MA, 1976.

BuUs S, Florentine M. Growth of loudness in listeners with cochlear hearing losses: recruitment reconsidered. J. Assoc. Res. Otolaryngol. 3:120-139, 2002.

CAI S. Intensity encoding of ventral cochlear nucleus neurons in normal and deafened cats and correlates of loudness recruitment. Unpublished Master's thesis. Department of Biomedical Engineering. The Johns Hopkins University, Baltimore, MD, 2007.

CANT NB, Morest DK. The structural basis for stimulus coding in the cochlear nucleus. In: Berlin CI (ed) Hearing Science: Recent Advances. London, College-Hill Press, 1984.

CARNEY LH. Sensitivities of cells in anteroventral cochlear nucleus of cat to spatiotemporal discharge patterns across primary afferents. J. Neurophysiol. 64:437-456, 1990.

CARNEY LH. Spatiotemproal encoding of sound level: models for normal encoding and recruitment of loudness. Hear. Res. 76:31-44, 1994.

Colburn HS, Carney LH, Heinz MG. Quantifying the information in auditory-nerve responses for level discrimination. J. Assoc. Res. Otolaryngol. 4:294-311, 2003.

Doucet JR, Ryugo DK. Structural and functional classes of multipolar cells in the ventral cochlear nucleus. Anat. Rec. Pt. A. 288:331-344, 2006.

Ertel JE, Fowlkes EB. Some algorithms for linear spline and piecewise multiple linear regression. J. Am. Stat. Assoc. 71:640648, 1976.
Evans EF. The sharpening of cochlear frequency selectivity in the normal and abnormal cochlear. Audiology. 14:419-442, 1975.

Ferragamo MJ, Golding NL, Oertel D. Synaptic inputs to stellate cells in the ventral cochlear nucleus. J. Neurophysiol. 79:51-63, 1998.

Francis HW, Manis PB. Effects of deafferetiation on the electrophysiology of ventral cochlear nucleus neurons. Hear. Res. 149:91105,2000

Golding NL, Robertson D, Oertel D. Recordings from slices indicate that octopus cells of the cochlear nucleus detect coincident firing of auditory nerve fibers with temporal precision. J. Neurosci. 15:138-3153, 1995.

HARrison RV. Rate-versus-intensity functions and related AP responses in normal and pathological guinea pig and human cochleas. J. Acoust. Soc. Am. 70:1036-1044, 1981.

HeInz MG, Young ED. Response growth with sound level in auditorynerve fibers after noise-induced hearing loss. J. Neurophysiol. 91:784-795, 2004.

Heinz MG, Issa JB, Young ED. Auditory-nerve rate responses are inconsistent with common hypotheses for the neural correlates of loudness recruitment. J. Assoc. Res. Otolaryngol. 6:91-105, 2005.

Hellman RP. Dependence of loudness growth on skirts of excitation patterns. J. Acoust. Soc. Am. 63:1114-1119, 1978.

Hellman RP, Zwislocki JJ. Loudness function of a 1000-cps tone in presence of a masking noise. J. Acoust. Soc. Am. 36:1618-1627, 1981.

IsaAcson JS, Walmsley B. Amplitude and time course of spontaneous and evoked excitatory postsynaptic currents in bushy cells of the anteroventral cochlear nucleus. J. Neurophysiol. 76:1566-1571, 1996.

JI, T. Representation of a Dynamic Speech Stream in the Cat Auditory Nerve. Unpublished Master's thesis. Department of Biomedical Engineering. Johns Hopkins University, Baltimore, MD, 2000.

JoHnson KO. Reconstruction of population response to a vibratory stimulus in quickly adapting mechanoreceptive afferent fiber population innervating glabrous skin of the monkey. J. Neurophysiol. 37:48-72, 1974.

Joris PX, Carney LH, SMith PH, Yin TCT. Enhancement of neural synchronization in the anteroventral cochlear nucleus. II: Responses in the tuning curve tail. J. Neurophysiol. 71:10371051, 1994.

Kiang NY, Moxon EC, Levine RA. Auditory-nerve activity in cats with normal and abnormal cochleas. In: Wolstenholme GEW, Knight T (eds) Sensorineural Hearing Loss. London, Churchill, pp. 241-273, 1970.

Kim JJ, Gross JS, Morest DK, Potashner SJ. Quantitative study of degeneration and new growth of axons and synaptic endings in the chinchilla cochlear nucleus after acoustic overstimulation. J. Neurosci. Res. 77:827-842, 2004.

Kopp-Scheinpflug C, Dehmel S, Dörrscheidt GJ, Rübsamen R. Interaction of excitation and inhibition in anteroventral cochlear nucleus neurons that receive large endbulb synaptic endings. J. Neurosci. 22:11004-11018, 2002.

Langers DRM, van Dijk P, Schoenmaker ES, Bakes WH. fMrI activation in relation to sound intensity and loudness. NeuroImage. 35:709-718, 2007.

Leao RN, Oleskevich SSH, Bautisa M, Fyffe Re, Walmsley B. Differences in glycinergic mIPSCs in the auditory brain stem of normal and congenitally deaf neonatal mice. J. Neurophysiol. 91:1006-1012, 2004.

Leao RN, Svahn K, Bernston A, Walmsley B. Hyperpolarizationactivated (I) currents in auditory brainstem neurons of normal and congenitally deaf mice. Eur. J. Neurosci. 22:147-157, 2005.

Leao RN, Sun H, Svahn K, Bernston A, Youssoufian M, Paolini AG, FYFFE RE, WALMSLey B. Topographic organization in the auditory brainstem of juvenile mice is disrupted in congenital deafness. J. Physiol. 571:563-578, 2006. 
Liberman MC. Single-neuron labeling and chronic cochlear pathology. I. Threshold shift and characteristic-frequency shift. Hear. Res. 16:33-41, 1984.

Liberman MC, Dodds LW. Single-neuron labeling and chronic cochlear pathology. III. Stereocilia damage and alterations of thresholds tuning curves. Hear. Res. 16:55-74, 1984.

Liberman MC, Kiang NY. Single-neuron labeling and chronic cochlear pathology. IV. Stereocilia damage and alterations in rate- and phase-level functions. Hear. Res. 16:75-90, 1984.

Lonsbury-Martin BL, Martin GK. Effects of moderately intense sound on auditory sensitivity in rhesus monkeys: behavioral and neural observations. J. Neurophysiol. 46:563-586, 1981.

Lu Y, Harris JA, RuBel EW. Development of spontaneous miniature EPSCs in mouse AVCN neurons during a critical period of afferentdependent neuron survival. J. Neurophysiol. 97:635-646, 2007.

MA W-L, Young ED. Loss of inhibition, and decreased spontaneous rates in dorsal cochlear nucleus following acoustic trauma. Hear. Res. 216-217:176-188, 2006.

Manis PB, Marx SO. Outward currents in isolated ventral cochlear nucleus neurons. J. Neurosci. 11:2865-2880, 1991.

May BJ, Le Prell GS, Sachs MB. Vowel representation in the ventral cochlear nucleus of the cat: effect of level, background noise, and behavioral state. J. Neurophysiol. 79:1755-1767, 1998.

Melcher JR. The cellular generators of brainstem auditory evoked potential. Unpublished doctoral thesis. Department of Electrical Engineering and Computer Science. Massachusetts Institute of Technology, Cambridge, MA, 1993.

Milbrandt JC, Holder TM, Wilson MC, Salvi RJ, Caspary DM. GAD levels and muscimol binding in rat inferior colliculus following acoustic trauma. Hear. Res. 147:251-260, 2000.

Miller RL, Schilding JR, Franck KR, Young ED. Effects of acoustic trauma on the representation of the vowel $/ \varepsilon /$ in cat auditory nerve fibers. J. Acoust. Soc. Am. 101:3602-3616, 1997.

MiskolczxFodor F. Relationship between loudness and duration of tonal pulses. III. Response in cases of abnormal loudness function. J. Acoust. Soc. Am. 32:486-492, 1960.

Moore BCJ. Perceptual Consequences of Cochlear Damage. New York, Oxford University Press, 2002.

Moore BCJ. Testing the concept of softness imperception: loudness near threshold for hearing-impaired ears. J. Acoust. Soc. Am. 115:3103-3111, 2004.

Moore BCJ, Glasberg BR. A revised model of loudness perception applied to cochlear hearing loss. Hear. Res. 188:70-88, 2004.

Moore BCJ, Glasberg BR, Hess RF, Birchall JP. Effects of flanking noise bands on the rate of growth of loudness of tones in normal and recruiting ears. J. Acoust. Soc. Am. 77:1505-1513, 1985.

Morita T, Naito Y, Nagamine T, Fujiki N, Shibasaki H, Ito J. Enhanced activation of the auditory cortex in patients with inner-ear hearing impairment: a magnetoencephalographic study. Clin. Neurophysiol. 114:851-859, 2003.

Mossop JE, Wilson MJ, Caspary DM, Moore DR. Down-regulation of inhibition following unilateral deafening. Hear. Res. 147:183187,2000 .

Muniak MA, Ray S, Hsiao SS, Dammann JF, Bensmaia SJ. The neural coding of stimulus intensity: Linking the population response of mechanoreceptive afferents with psychophysical behavior. J. Neurosci. 27:11687-11699, 2007.

Nelson JJ, Chen K. The relationship of tinnitus, hyperacusis, and hearing loss. Ear Nose Throat J. 83:472-476, 2004.

Oertel D. Synaptic repsonses and electrical properties of cells in brain slices of the mouse anteroventral cochlear nucleus. J. Neurosci. 3:2043-2053, 1983.

OERTEL D. Use of brain slices in the study of the auditory system: Spatial and temporal summation of synaptic inputs in cells in the anteroventral cochlear nucleus of the mouse. J. Acoust. Soc. Am. 78:328-333, 1985.
Oleskevich S, Walmsley B. Synaptic transmission in the auditory brainstem of normal and congenitally deaf mice. J. Physiol. 540:447-455, 2002.

OsEN KK. Course and termination of primary afferents in the cochlear nuclei of the cat. An experimental study. Arch. Ital. Biol. 108:21-51, 1970.

Palmer AR, Evans EF. Intensity coding in the auditory periphery of the cat: responses of cochlear nerve and cochlear nucleus neurons to signals in the presence of bandstop masking noise. Hear. Res. 7:305-323, 1982.

PfeIFFER RR. Anteroventral cochlear nucleus: wave forms of extracellularly recorded spike potentials. Science 154:667-668, 1966.

PFEIFFER RR, KIM DO. Cochlear nerve fiber responses: distribution along the cochlear partition. J. Acoust. Soc. Am. 58:867-869, 1975.

PickLes JO. Psychophysical frequency resolution in the cat as determined by simultaneous masking and its relation to auditory-nerve resolution. J. Acoust. Soc. Am. 66:1725-1732, 1979.

PICKLEs JO. Auditory-nerve correlates of loudness summation with stimulus bandwidth, in normal and pathological cochleae. Hear. Res. 12:239-250, 1983.

POPElÁr̆ J, SYKa J, Berndt H. Effect of noise on auditory evoked responses in awake guinea pigs. Hear. Res. 26:239-247, 1987.

Pugh JE JR, Moody DB, Anderson DJ. Electrocochleography and experimentally induced loudness recruitment. Eur. Arch. Otorhino-laryngol. 224:241-255, 1979.

Qiu C, Salvi RJ, Ding D, Burkard R. Inner hair cell loss leads to enhanced response amplitudes in auditory cortex of unanesthetized chinchillas: evidence for increased system gain. Hear. Res. 139:153-171, 2000.

Raman IM, Trussell LO. The kinetics of the response to glutamate and kainate in neurons of the avian cochlear nucleus. Neuron 9:173-186, 1992.

Redd EE, Pongstaporn T, Ryugo DK. The effects of congenital deafness on auditory nerve synapses and globular bushy cells in cats. Hear. Res. 147:160-174, 2000.

Redd Ee, Cahill HB, Pongstapron T, Ryugo DK. The effects of congenital deafness on auditory nerve synapses: Type I and Type II multipolar cells in the anteroventral cochlear nucleus of cats. J. Assoc. Res. Otolaryngol. 3:403-417, 2002.

ReLkin EM, Doucet JR. Is loudness simply proportional to the auditory nerve spike count? J. Acoust. Soc. Am. 101:2735-2740, 1997.

Rhode WS, GreEnberg S. Lateral suppression and inhibition in the cochlear nucleus of the cat. J. Neurophysiol. 71:493-514, 1994.

Rhode WS, Sмiтн PH. Encoding timing and intensity in the ventral cochlear nucleus of the cat. J. Neurophysiol. 56:261-286, 1986.

Rhode WS, Oertel D, Smith PH. Physiological response properties of cells labelled intracellularly with horseradish peroxide in cat ventral cochlear nucleus. J. Comp. Neurol. 213:448-463, 1983.

Rothman JS, Manis PB. Differential expression of three distinct potassium currents in the ventral cochlear nucleus. J. Neurophysiol. 89:3070-3082, 2003.

Rothman JS, Young ED. Enhancement of neural synchronization in computational models of ventral cochlear nucleus bushy cells. Audit. Neurosci. 2:47-62, 1996.

Ruggero MA, Rich NC. Furosemide alters organ of corti mechanics: evidence for feedback of outer hair cells upon the basilar membrane. J. Neurosci. 11:1057-1067, 1991.

Ryugo DK. The auditory nerve: peripheral innervation, cell body morphology, and central projections. In: Webster DB, Popper AN, Fay RR (eds) The Mammalian Auditory Pathway: Neuroanatomy. New York, Springer, 1992.

Ryugo DK, Pongstapron T, Huchton DM, NIPARKo JK. Ultrastructural analysis of primary endings in deaf white cats: morphologic alterations in endbulbs of Held. J. Comp. Neurol. 385:230-244, 1997. 
SACHS MB, ABbas PJ. Rate versus level functions for auditory nerve fibers in cats: tone-burst stimuli. J. Acoust. Soc. Am. 56:1835-1847, 1974.

SAchs MB, Young ED. Encoding of steady-state vowels in the auditory nerve: representation in terms of discharge rate. J. Acoust. Soc. Am. 66:470-479, 1979.

Salvi RJ, Hamernik RP, Henderson D. Response patterns of auditory nerve fibers during temporary threshold shift. Hear. Res. 10:3767, 1983.

Salvi RJ, Saunders JC, Gratton MA, Arehole S, Powers N. Enhanced evoked response amplitudes in the inferior colliculus of the chinchilla following acoustic trauma. Hear. Res. 50:245-258, 1990.

SALVI RJ, WANG J, Ding D. Auditory plasticity and hyperactivity following cochlear damage. Hear. Res. 147:261-274, 2000.

Saunders JC, Bock GR, James R, Chen C-S. Effects of priming for audiogenic seizure on auditory evoked responses in the cochlear nucleus and inferior colliculus of BALB/c mice. Exp. Neurol. 37:388-394, 1972.

Schaette R, Kempter R. Development of tinnitus-related neuronal hyperactivity through homeostatic plasticity after hearing loss: a computational model. Eur. J. Neurosci. 143:103-109, 2006.

SEWELL WF. Furosemide selectively reduces one component in rate-level functions from auditory-nerve fibers. Hear. Res. 15:69-72, 1984.

SHerlock LP, Formby C. Estimates of loudness, loudness discomfort, and the auditory dynamic range: normative estimates, comparison of procedures, and test-retest reliability. J. Am. Acad. Audiol. 16:85-100, 2005.

Sмrтн RL. Encoding sound intensity by auditory neurons. In: Edelman GM, Gall WE, Cowan WM (eds) Auditory Function: Neurobiological Bases of Hearing. New York, Wiley, pp. 243-274, 1988.

SмITH PH, Rhode WS. Characterization of HRP-labeled globular bushy cells in the cat anteroventral cochlear nucleus. J. Comp. Neurol. 266:360-375, 1987.

Sмith PH, Rhode WS. Structural and functional properties distinguish two types of multipolar cells in the ventral cochlear nucleus. J. Comp. Neurol. 282:595-616, 1989.

Stillman JA, Znislocki JJ, Zhang M, Cefaratti LK. Intensity justnoticeable differences at equal-loudness levels in normal and pathological ears. J. Acoust. Soc. Am. 93:425-434, 1993.

Suneja SK, Benson CG, Potashner SJ. Glycine receptors in adult guinea pig brain stem auditory nuclei: regulation after unilateral cochlear ablation. Exp. Neurol. 154:473-488, 1998.
Syka J, Rybalko N, Popelář J. Enhancement of the auditory cortex evoked responses in awake guinea pigs after noise exposure. Hear. Res. 78:158-168, 1994.

Szczepaniak WS, Møller AR. Evidence of neuronal plasticity within the inferior colliculus after noise exposure: a study of evoked potentials in the rat. Electroencephalogr. Clin. Neurophysiol. 100:158-164, 1996.

Vale C, Sanes DH. Afferent regulation of inhibitory synaptic transmission in the developing auditory midbrain. J. Neurosci. 20:1912-1921, 2000.

Vale C, Schoorlemmer J, Sanes DH. Deafness disrupts chloride transporter function and inhibitory synaptic transmission. J. Neurosci. 23:7516-7524, 2003.

Vale C, Júz JM, Moore DR, SANes DH. Unilateral cochlear ablation produces greater loss of inhibition in the contralateral inferior colliculus. Eur. J. Neurosci. 20:2133-2140, 2004.

Wang Y, Manis PB. Synaptic transmission at the cochlear nucleus endbulb synapse during age-related hearing loss in mice. J. Neurophysiol. 94:1814-1824, 2005.

WANG J, Ding D, SaLVI RJ. Functional reorganization in chinchilla inferior colliculus associated with chronic and acute cochlear damage. Hear. Res. 168:238-249, 2002.

Willott JF, Milbrandt JC, Bross LS, Caspary DM. Glycine immunoreactivity and receptor binding in the cochlear nucleus of C57BL/6J and CBA/CaJ mice: effects of cochlear impairment and aging. J. Comp. Neurol. 385:405-414, 1997.

Winter IM, Robertson D, Yates GK. Diversity of characteristics frequency-rate-intensity functions in guinea pig auditory nerve fibers. Hear Res 45:191-202, 1990.

Young ED, Oertel D. Cochlear nucleus. In: Sheperd GM (ed) The Synaptic Organization of the Brain. New York, Oxford University Press, 2004.

Young ED, SACHS MB. Auditory nerve inputs to cochlear nucleus neurons studied with cross-correlation. Neuroscience. 154:127138,2008

Young ED, Robert JM, Shofner MP. Regularity and latency of units in ventral cochlear nucleus: implications for unit classification and generation of response properties. J. Neurophysiol. 60:1-29, 1988.

Zeng F-G, Turner CW. Binaural loudness matches in unilaterally impaired listeners. Quart. J. Exp. Psychol. 43A:565-583, 1991. 